\title{
White matter connections of high-level visual areas predict cytoarchitecture better than category-selectivity
}

Emily Kubota ${ }^{1 *}$, Mareike Grotheer ${ }^{2,3}$, Dawn Finzi ${ }^{1}$, Vaidehi S. Natu ${ }^{1}$, Jesse Gomez ${ }^{4}$, and Kalanit Grill-Spector ${ }^{1,5,6}$

1. Department of Psychology, Stanford University, Stanford, CA

2. Department of Psychology, University of Marburg, Marburg 35039, Germany.

3. Center for Mind, Brain and Behavior - CMBB, Philipps-Universität Marburg and Justus-Liebig-Universität Giessen, Germany

4. Princeton Neuroscience Institute, Princeton University, NJ

5. Neurosciences Program, Stanford University, Stanford, CA

6. Wu Tsai Neurosciences Institute, Stanford University, Stanford, CA

*Corresponding Author: ekubota@stanford.edu 


\begin{abstract}
Ventral temporal cortex (VTC) consists of high-level visual regions that are arranged in consistent anatomical locations across individuals. This consistency has led to several hypotheses about the factors that constrain the functional organization of VTC. A prevailing theory is that white matter connections influence the organization of VTC, however, the nature of this constraint is unclear. Here, we test two hypotheses: (1) white matter tracts are specific for each category or (2) white matter tracts are specific to cytoarchitectonic areas of VTC. To test these hypotheses, we used diffusion magnetic resonance imaging $(\mathrm{dMRI})$ to identify white matter tracts and functional magnetic resonance imaging (fMRI) to identify category-selective regions in VTC in children and adults. We find that in childhood, white matter connections are linked to cytoarchitecture rather than category-selectivity. In adulthood, however, white matter connections are linked to both cytoarchitecture and category-selectivity. These results suggest a rethinking of the view that category-selective regions in VTC have category-specific white matter connections early in development. Instead, these findings suggest that the neural hardware underlying the processing of categorical stimuli may be more domain-general than previously thought, particularly in childhood.
\end{abstract}

Keywords: development, fusiform face area, ventral stream, visual word form area 


\section{Introduction}

Ventral temporal cortex (VTC) consists of regions that respond more strongly to categories of stimuli such as faces (Kanwisher et al., 1997), places (Epstein \& Kanwisher, 1998), body parts (Peelen \& Downing, 2005), and words (Cohen et al., 2003) than any other stimulus (referred to as category-selective regions (Kanwisher, 2010)). These regions fall in consistent anatomical locations across individuals (Grill-Spector \& Weiner, 2014; Weiner et al., 2014, 2018), which has led researchers to ask which factors constrain this functional organization. Classic theories suggest that function, cytoarchitecture, and connectivity all contribute to cortical organization (Amunts \& Zilles, 2015; Van Essen et al., 1992; Zeki \& Shipp, 1988). While past work has investigated the relationship between white matter and category-selectivity (Bouhali et al., 2014; Gomez et al., 2015; Grotheer et al., 2019; Grotheer, Yeatman, et al., 2021; Gschwind et al., 2012; Li et al., 2020; Osher et al., 2016; Pyles et al., 2013; Saygin et al., 2011, 2016; Thomas et al., 2009; Yeatman et al., 2013) cytoarchitecture and category-selectivity (Weiner, Barnett, et al., 2017), and white matter and cytoarchitecture (Johansen-Berg et al., 2004; Klein et al., 2007), there has been limited research on the interplay between all three factors, which is the focus of the present study.

To investigate the relationship between white matter, cytoarchitecture, and category-selectivity, we use word- (mOTS-words, pOTS-words) and face-selective (pFusfaces and mFus-faces) functional regions of interest (ROIs) in VTC as a model system (Figure 1A). These regions are an excellent model system as mFus-faces and mOTSwords are located within the same cytoarchitectonic area, FG4, but are selective for different categories, faces and words, respectively (Weiner, Barnett, et al., 2017). pFusfaces and pOTS-words are also located within the same cytoarchitectonic area, this time FG2, and are selective for words and faces, respectively (Weiner, Barnett, et al., 2017). That is, mOTS-words and pOTS-words are both selective for words, but are located within different cytoarchitectonic areas, and mFus-faces and pFus-faces are both selective for faces, but are located within different cytoarchitectonic areas. Notably, categoryselectivity and cytoarchitecture are orthogonal in this case, allowing us to ask whether white matter connections align with category-selectivity, cytoarchitecture, or both. 
Previous studies provide support for the hypothesis that white matter connections of VTC functional regions are organized by category-selectivity. For example, studies examining the white matter fingerprint of VTC have shown that category-selective responses can be predicted by white matter connectivity to the rest of the brain (Li et al., 2020; Osher et al., 2016; Saygin et al., 2011, 2016). It has been hypothesized that different white matter connections of word- and face-selective regions may support the specific processing demands associated with the region's preferred category. For example, word-selective regions may have more connections to language regions than face-selective regions to support the task of reading (Bouhali et al., 2014). This hypothesis aligns with the theory that white matter tracts might designate specific parts of cortex for processing different categories, perhaps from birth (Li et al., 2020). Supporting this view, researchers found that (i) congenitally blind participants have a similar functional organization of VTC (Murty et al., 2020; van den Hurk et al., 2017) as well as similar functional connectivity to early visual cortex as sighted participants (Butt et al., 2013; Striem-Amit et al., 2015), suggesting that there are innate anatomical constraints determining cortical organization (Mahon \& Caramazza, 2011), and (ii) the white matter fingerprint of VTC at age five can predict where word-selective cortex will emerge at age eight (Saygin et al., 2016). These studies suggest that white matter might be an innate or early developing constraint on category-selective processing. Moreover, these studies suggest that functional regions selective for the same category will have similar white matter connectivity to support category-specific processing.

Alternatively, classical investigations in human (Flechsig, 1920; Zilles, 2018) and nonhuman primates (Van Essen et al., 1992; Zeki \& Shipp, 1988) suggest that white matter connections may align with cytoarchitecture. For example, past work has shown that subregions of the frontal lobe with distinct cytoarchitecture also have distinct white matter connections (Johansen-Berg et al., 2004; Klein et al., 2007). Since the time of Brodmann, it has also been suggested that cytoarchitectonic areas might correspond to functional regions (Zilles, 2018). However, recent work has demonstrated that this view is overly simplistic and there is not always a one-to-one mapping between cytoarchitecture and functional regions (Glasser et al., 2016; Rosenke et al., 2018; Weiner, Barnett, et al., 2017). For example, a single cytoarchitectonic area in VTC, FG4, 
contains regions selective for three categories: faces, words, and body parts (Weiner, Barnett, et al., 2017). Therefore, these studies raise the possibility that functional regions located within the same cytoarchitectonic area might have similar white matter connectivity irrespective of category-selectivity.

In addition to the debate on whether white matter connections of VTC functional ROIs are category-specific or cytoarchitectonic-specific, a related question is whether white matter connections of VTC are fixed across development or if they change with the functional development of VTC during childhood. Indeed, while some functional regions, like face-selective regions may be present in infancy (Deen et al., 2017; Kosakowski et al., 2021), other regions, such as word-selective regions, emerge later, only when children learn to read (Brem et al., 2010; Dehaene-Lambertz et al., 2018; Kubota et al., 2019). Notably, both word- and face-selective regions continue to develop well into adolescence (Golarai et al., 2010; Nordt et al., 2021). We reason that if white matter connections lay an intrinsic blueprint for later development of category-selectivity (Li et al., 2020; Saygin et al., 2016), then the white matter connections of VTC functional ROls will be consistent across development. Alternatively, as category-selective regions continue to develop from childhood into adolescence, there may be a dynamic interplay between the development of functional regions and their white matter connectivity. This hypothesis predicts that white matter connections of face- and word-selective regions will change from childhood to adulthood.

To determine the nature of VTC white matter connections and their development, we collected functional magnetic resonance imaging (fMRI) and diffusion magnetic resonance imaging (dMRI) in 27 children (ages $5.76-12.39$ years, mean \pm sd: $9.74 \pm 2.11$

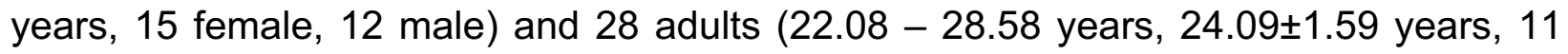
female, 17 male). We used a fMRI localizer experiment (Stigliani et al., 2015) to define functional ROIs in each individual's brain and dMRI data to generate a whole brain white matter connectome for each individual. We intersected each participant's whole brain connectome with each of their functional ROls to identify the functionally defined white matter associated with each functional ROI (Finzi et al., 2021; Grotheer et al., 2019; Grotheer, Yeatman, et al., 2021). We then tested if the functionally defined white matter of face (pFus- and mFus-faces) and word-selective regions (pOTS- and mOTS-words) is 
linked to category, cytoarchitecture, or both, and if they change from childhood to adulthood.

\section{Materials and Methods}

\section{Participants}

The study was approved by the Institutional Review Board of Stanford University. Prior to the start of the study, all participants gave written consent. 29 children and 28 adults participated in the experiment. One child was excluded for an incomplete dataset, and one child was excluded due to a cyst on the anterior temporal lobe. Thus, we report data from $\mathrm{N}=27$ children (ages $5.76-12.39$ years $(\mathrm{M}=9.74, \mathrm{SD}=2.11)$, 15 female, 12 male), and $\mathrm{N}=28$ adults (ages $22.08-28.58$ years $(\mathrm{M}=24.09, \mathrm{SD}=1.59), 11$ female, 17 male). The racial demographics of the sample were as follows: 56\% White, 15\% Asian, 9\% mixed-race, $7 \%$ Hispanic, 5\% Black, $4 \%$ other, $4 \%$ missing data.

\section{MRI}

MRI data were collected at the Center for Cognitive and Neurobiological Imaging at Stanford University, using a GE 3 Tesla Signa Scanner with a 32-channel head coil.

\section{Anatomical MRI}

We acquired quantitative MRI measurements using protocols from Mezer et al. (2013) to generate a $1 \mathrm{~mm}$ isotropic T1-weighted anatomical image of each participant. Anatomical images were used for gray matter/white matter segmentation, cortical surface reconstruction, and visualization of data on the cortical surface.

\section{Diffusion MRI (dMRI) acquisition}

Diffusion-weighted dMRI was acquired using a dual-spin echo sequence in 96 different directions, 8 non-diffusion-weighted $(b=0)$ images were collected, 60 slices provided full head coverage. Resolution $=2 \mathrm{~mm}$ isotropic, $\mathrm{TR}=8000 \mathrm{~ms}$, TE $=93.6 \mathrm{~ms}$, FOV=220mm, flip angle $\left.=90^{\circ}, \mathrm{b} 0=2000 \mathrm{~s} / \mathrm{mm}^{2}\right)$. 


\section{Functional MRI acquisition}

We acquired simultaneous multi-slice, one-shot $\mathrm{T}^{*}$ sensitive gradient echo EPI sequences with a multiplexing factor of 3 and near whole brain coverage (48 slices), $\mathrm{FOV}=192 \mathrm{~mm}, \mathrm{TR}=1 \mathrm{~s}, \mathrm{TE}=30 \mathrm{~ms}$, and flip angle $=76^{\circ}$. Resolution $=2.4 \mathrm{~mm}$ isotropic.

Functional localizer task: Participants took part in a 10-category functional localizer experiment (Nordt et al., 2021; Stigliani et al., 2015). The stimuli included faces (children, adults), characters (pseudowords, numbers), bodies (headless bodies, limbs), places (houses, corridors), and objects (cars, string instruments). Stimuli were presented in 4 second blocks with $2 \mathrm{~Hz}$ stimulus presentation rate. Participants were instructed to fixate throughout the experiment and complete an oddball task, which required pressing a button whenever a phase-scrambled image appeared. Each run was 5 minutes and 18 seconds long and participants completed 3 runs of the experiment.

\section{Data Analyses}

Diffusion MRI processing

dMRI data were preprocessed using MRtrix3 (Tournier et al., 2019; https://github.com/MRtrix3/mrtrix3) and mrDiffusion (http://github.com/vistalab) as in past work (Grotheer et al., 2019; https://github.com/VPNL/fat). Data were denoised using principal component analysis, Rician based denoising, and Gibbs ringing corrections. Eddy currents and motion were corrected for using FSL (https://fsl.fmrib.ox.ac.uk/), and bias correction was performed using ANTs. dMRI data were aligned to the b0 images and then aligned to the high-resolution T1-weighted image of the same brain. Voxel-wise fiber orientation distributions (FOD) were calculated using constrained spherical deconvolution (CSD) (Tournier et al., 2007).

\section{Exclusion criterion}

dMRI data with more than $5 \%$ outliers were excluded. All participants were included under this threshold. 
Generating white matter connectomes: We used MRtrix3 to generate five candidate connectomes with the following maximum angles $\left(2.25^{\circ}, 4.5^{\circ}, 9^{\circ}, 11.25^{\circ}\right.$, and $\left.13.5^{\circ}\right)$. The goal of using multiple candidate connectomes was to avoid biasing the results towards either long range or local connections (Takemura et al., 2016). For each candidate connectome we used probabilistic tracking with the IFOD1 algorithm, a step size of 0.2 $\mathrm{mm}$, a minimum length of $4 \mathrm{~mm}$, a maximum length of $200 \mathrm{~mm}$, and a FOD amplitude stopping criterion of 0.1 . Each candidate connectome consisted of 500,000 streamlines. We used anatomically constrained tractography (ACT; Smith et al., 2012) to identify the gray-matter white-matter interface directly below the functional ROIs. Streamlines were seeded on this interface to ensure that they reached the gray-matter white-matter interface. This is critical for accurately identifying the connections that reach functional ROls, which are located in the gray matter (Grotheer, Kubota, et al., 2021). Finally, we concatenated the candidate connectomes into a single connectome containing 2,500,000 streamlines. We refer to this as the white matter connectome of each subject.

To identify the main white matter bundles (fascicles) of the brain we used Automated Fiber Quantification (Yeatman et al., 2012) (AFQ; https://github.com/yeatmanlab/AFQ). The output of AFQ are 11 fascicles in each hemisphere and 2 cross-hemispheric fascicles.

\section{fMRI processing}

Functional data was aligned to the anatomical brain volume of each individual subject. Motion correction was performed both within and across runs. No spatial smoothing or slice-timing correction was performed. To estimate the response to each category a general linear model (GLM) was fit to each voxel by convolving the stimulus presentation with the hemodynamic response function.

\section{Exclusion criterion}

Criteria for exclusion of data were within-run motion $>2$ voxels or between-run motion >3 voxels. All participants were included under this threshold. 


\section{Functional region of interest definition}

To identify a functional ROI that showed significant selectivity to exemplars of a particular category, we used a contrast comparing the response to the category of interest compared to the other categories and included voxels that passed the statistical threshold ( $\mathrm{t}>3$, voxel level uncorrected). For word-selective regions mOTS-words and pOTSwords, we contrasted words $>$ all other categories (excluding numbers). For faces we contrasted adult and child faces $>$ all others. We defined the functional ROls on the inflated cortical surface of each participant to ensure the correct anatomical location of each functional ROI.

\section{Connectivity profiles of functionally defined white matter}

To identify the white matter associated with each functional region we intersected each functional ROI (mOTS-words, mFus-faces, pOTS-words, pFus-faces; defined from the fMRI data) with the white matter connectome, the product of which we refer to as functionally defined white matter. All analyses were done in the native brain space of each subject. For each functional ROI we defined a connectivity profile in two ways: (1) its fascicle connectivity profile, and (2) its endpoint connectivity profile.

(1) Fascicle connectivity profile: To identify the fascicles connected to each functional ROI, we intersected the subject's fascicles classified by AFQ with the gray matter/white matter interface directly adjacent to that functional ROI (see (Finzi et al., 2021; Grotheer et al., 2019; Grotheer, Kubota, et al., 2021). This process gave us the functionally defined white matter fascicles for each functional ROI. Previous work showed that out of those fascicles identified by AFQ, only 5 fascicles connect to VTC: the inferior longitudinal fasciculus, inferior frontal occipital fasciculus, arcuate fasciculus, posterior arcuate fasciculus, and vertical occipital fasciculus (Ben-Shachar et al., 2007; Broce et al., 2019; Gomez et al., 2015; Grotheer et al., 2019; Grotheer, Yeatman, et al., 2021; Kay \& Yeatman, 2017; Lerma-Usabiaga et al., 2018; Song et al., 2015; Tavor et al., 2014; Thomas et al., 2009; Wandell \& Yeatman, 2013; Weiner et al., 2016; Yeatman et al., 2013). To determine the fascicle connectivity profile, we the calculated the distribution of its connections across these five fascicles for each functional $\mathrm{ROI}$ in each subject. That 
is, the proportion of the functionally defined white matter fascicles associated with each of these particular fascicles.

(2) Endpoint connectivity profile: To identify the endpoints associated with each functional ROI, we intersected the whole brain connectome of each subject with the gray matter/white matter interface directly adjacent to the functional ROI. This approach identifies all white matter tracts that intersect with the functional ROI and not only those which belong to the large fascicles. We projected the endpoints of the functionally defined white matter to the cortical surface using tract density imaging (TDI) with mrTrix3 (Tournier et al., 2019) and calculated the distribution of endpoints of these tracts across all cortical surface vertices. We transformed the TDI output into a distribution of endpoints by dividing the endpoint map by the total number of endpoints. This results in an endpoint density map that sums to one for each functional ROI and subject. To quantify the endpoint density map in an interpretable manner that is related to a common whole brain atlas, we measured the average endpoint density in each of the 180 (per hemisphere) Glasser Atlas ROls (Glasser et al., 2016). Thus, we projected the Glasser Atlas from the fsaverage cortical surface into each participant's native cortical brain surface using cortex-based alignment in FreeSurfer version 6.0, and then calculated in each subject the average endpoint density in each of the Glasser ROls. We also calculated the average endpoint connectivity density maps across participants by projecting each individual's subject map to the fsaverage cortical surface using cortex-based alignment and averaging across participants.

Evaluating similarity between connectivity profiles: We used a correlation metric to calculate the similarity between connectivity profiles of pairs of functional ROls.

Predicting cortical parcellations from connectivity profiles: To test whether connectivity profiles predict the cytoarchitectonic area, the category-selectivity of a functional ROI, or both, we used a winner-takes-all maximum correlation classifier using a leave-one-out cross-validation procedure. All three classification types were performed for both the fascicle connectivity profiles and the endpoint connectivity profiles. 
a) Cytoarchitecture classification (2-way classification): Training: We calculated the average connectivity profile of functional ROls in each cytoarchitectonic area (FG2 and FG4), excluding the held-out participant's connectivity profiles. Testing: We classified the cytoarchitectonic area of each of the unlabeled, held-out connectivity profiles based on the highest correlation with the connectivity profiles of the training set. That is, the held-out connectivity profile was classified as belonging to the cytoarchitectonic area with which it was most highly correlated. This classification was then compared to the true cytoarchitectonic area to determine the accuracy of the classifier. We repeated this procedure across all n-folds for the connectivity profile of each functional ROI.

b) Category classification (2-way classification): Training: We calculated the average connectivity profile of functional ROls with the same category-selectivity (words and faces), excluding the held-out participant's connectivity profiles. Testing: We classified the cytoarchitectonic area of each of the unlabeled, held-out connectivity profiles based on the highest correlation with the training connectivity profiles. This classification was then compared to the true category-selectivity to determine the accuracy of the classifier. We repeated this procedure across all n-folds for the connectivity profile of each functional ROI.

c) Functional ROI classification (4-way classification): Training: We calculated the average connectivity profile for each functional ROI (mFus-faces/mOTSwords/pFus-faces/pOTS-words) excluding the held-out participant's connectivity profiles. Testing: We classified the connectivity profile of the held-out functional $\mathrm{ROI}$ based on the highest correlation with the training connectivity profiles. This classification was then compared to the true functional ROI label to determine the accuracy of the classifier. We then repeated the procedure for each connectivity profile of each functional ROI across all n-folds.

\section{Controls for development differences}

As we found developmental differences, we further examined whether between agegroup differences in motion, functional $\mathrm{ROI}$ size, or functional ROI location could drive the developmental differences in connectivity profiles across children and adults. 


\section{Motion control}

Even though all subjects met our inclusion criterion, both rotation and translation were significantly different between children and adults (rotation: $t(53)=-2.63, p=0.01$, translation: $t(53)=-2.24, p=0.03$. To control for this difference, we repeated the analyses comparing across age-groups (connectivity profile similarity analysis and classification analysis) using the subset of participants that were matched across ages for motion. This was achieved by excluding 5 participants that had either the most rotation $(n=4)$ or translation ( $n=2$, one overlapping with rotation). The excluded participants were: one six-year-old, one seven-year-old, two nine-year-olds, and one eleven-year-old. After excluding these participants there were no longer significant differences in rotation $(t(48)$ $=-1.67, p=0.10)$ and translation $(t(48)=-0.98, p=0.33)$ across children and adults.

\section{Constant size ROI control}

For the main analyses, we use functional ROls defined from contrasts in each subject's brain as outlined above because it is the most accurate way to define functional ROls for each participant. However, if there are developmental changes in ROls sizes they may affect connectivity profiles across age groups. Therefore, as a control, we repeated both fascicle and endpoint connectivity analyses using a constant sized, 3mm disk ROI centered at the center of each functional ROI on each subject's native brain.

\section{VTC exclusion control}

For the main analyses of white matter endpoints, we examined all endpoints across the entire brain as this reflects the entirety of the connectivity endpoint maps. However, as some of these endpoints are in VTC, they may be particularly affected by between age group differences in the location and spatial extent of functional ROls within VTC. To further test that development of endpoint connectivity is not just due to developmental differences restricted to VTC, we repeated connectivity endpoints analyses excluding 11 Glasser ROls that overlap with VTC (see Nordt et al., 2019): PHA1, PHA3, TE2p, PH, PIT, FFC, V8, VVC, VMV2, VMV3, TF. 


\section{Statistics}

In general, statistical analyses were computed using the ImerTest (Kuznetsova et al., 2017) package in R. Linear mixed-effects models (LMMs) were used because our data include missing data points (not all participants have all functional ROls). The significance of LMM model terms was evaluated using repeated-measures analyses-of-variance (LMM ANOVAs; type III) with Satterthwaite's method of correction for degrees of freedom (Luke, 2017).

Testing for significant differences in similarity among connectivity profiles: We measured the pairwise correlation between connectivity profiles to quantify their similarity. To test whether connectivity profiles were more correlated for functional ROls located within the same cytoarchitectonic area or selective for the same category and if this differed by age group, we used the following model:

Correlation shared_feature(cytoarchitecture/category) * group (children/adults) + (1|subject).

Testing if classification accuracy is significantly above chance: To test whether classification accuracy was above chance, we performed a one-sample $t$-test testing whether classification was significantly different than the chance level $(50 \%$ for cytoarchitecture/category classification; $25 \%$ for functional ROI classification).

Testing for significant differences in classification accuracy: To test whether classification accuracy differed by classification type (cytoarchitecture or category) and age group we used the following model:

Classification accuracy $\sim$ classification feature (cytoarchitecture/category) ${ }^{*}$ group (children/adults) $+(1 \mid$ subject $)$.

\section{Results}

We examined the functionally defined white matter for face- and word-selective VTC ROls, using two complementary approaches. In the first approach, we identified the 
fascicles (Catani et al., 2002; Yeatman et al., 2012) that connect to each functional ROI, and calculated the distribution of connections across the fascicles. We refer to this quantification as the fascicle connectivity profile of each functional region. The fascicle connectivity profile provides an interpretable quantification of the white matter connections of each functional ROI because the anatomical layout of the major fascicles is well-established (Catani et al., 2002; Wakana et al., 2004; Yeatman et al., 2012). In the second approach, which we refer to as the endpoint connectivity profile, we determined the distribution of endpoints of the white matter connections of each functional ROI across the entire cortex.

Fascicle connectivity profiles of VTC functional ROIs are organized by cytoarchitecture in children \& adults

We first examined the fascicle connectivity profiles of VTC face- and wordselective regions in each individual's brain. We were able to identify all fascicles in all participants and functional ROls in most participants (children: mFus-faces: $n=22$, mOTS-words: $\mathrm{n}=20$, pFus-faces, $\mathrm{n}=20$, pOTS-words: $\mathrm{n}=17$; adults: mFus-faces: $\mathrm{n}=$ 26, mOTS-words: $n=25$, pFus-faces, $n=20$, pOTS-words: $n=26$ ).

There are five fascicles that connect to visual regions in VTC (Ben-Shachar et al., 2007; Broce et al., 2019; Gomez et al., 2015; Grotheer et al., 2019; Grotheer, Yeatman, et al., 2021; Kay \& Yeatman, 2017; Lerma-Usabiaga et al., 2018; Song et al., 2015; Tavor et al., 2014; Thomas et al., 2009; Wandell \& Yeatman, 2013; Weiner et al., 2016; Yeatman et al., 2013) (Fig 1B-top): the inferior frontal occipital fasciculus (IFOF) (Catani et al., 2002), which connects the occipital lobe and frontal lobe, the inferior longitudinal fasciculus (ILF) (Catani et al., 2002), which connects the occipital lobe and ventral temporal lobe, the arcuate fasciculus (AF) (Catani et al., 2002), which connects the ventral temporal lobe and the frontal lobe, the posterior arcuate fasciculus (pAF) (Weiner, Yeatman, et al., 2017), which connects the ventral temporal lobe and the parietal lobe, and the vertical occipital fasciculus (VOF) (Takemura et al., 2016; Weiner, Yeatman, et al., 2017; Yeatman et al., 2014), which connects the ventral and dorsal occipital lobe. Therefore, our fascicle connectivity analysis will focus on these five fascicles. The connectivity profiles are quantified as the percentage of tracts that connect to each of 
these five fascicles (see Fig 1B-bottom). Since word-selective regions are left lateralized (Petersen et al., 1988), left hemisphere data are provided in the main figures and right hemisphere data are presented in the Supplemental figures.
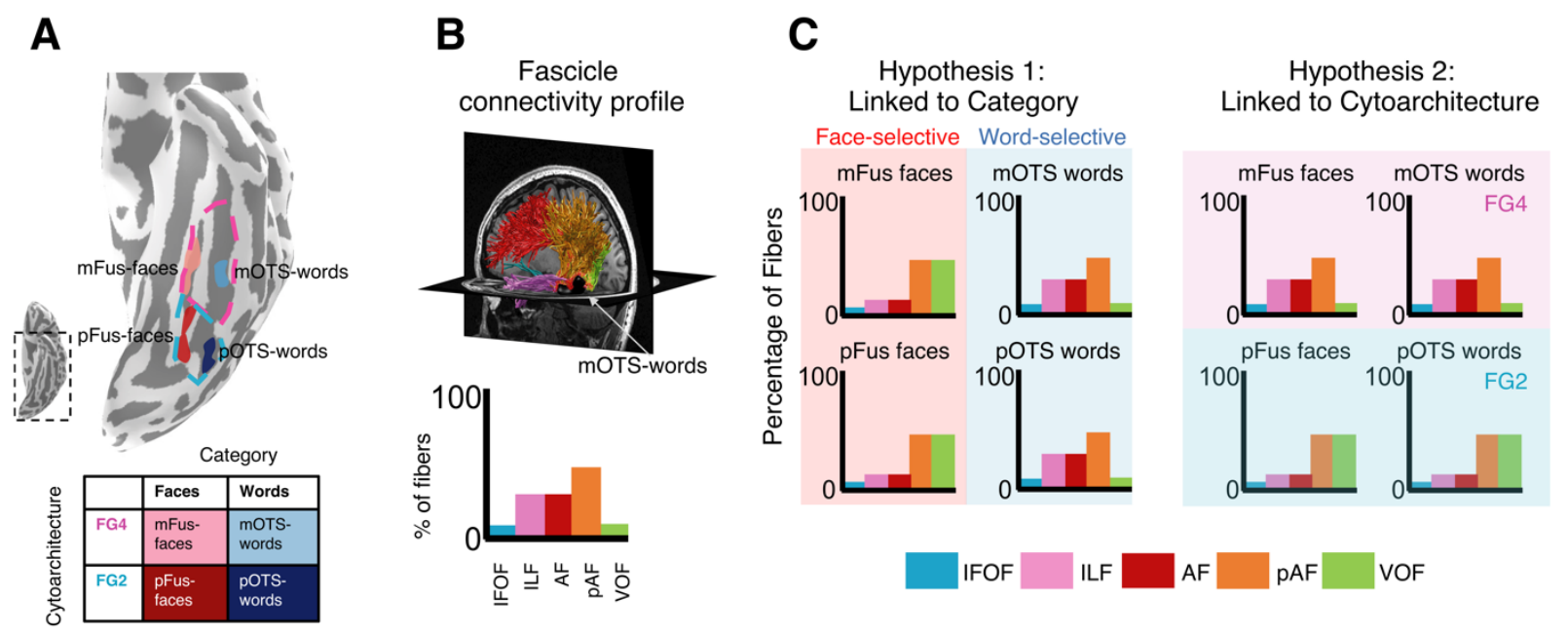

Figure 1. Design and hypothesis. (A) Word and face-selective functional ROIs provide a model system to test our experimental hypotheses. The schematic shows how face-selective (reds) and word-selective (blues) regions are organized relative to cytoarchitecture (rows) and category (columns). The inflated left hemisphere shows the face-selective (reds) and word-selective (blues) regions on the cortical surface. The cytoarchitectonic areas are depicted in the dashed outlines (FG4: magenta; FG2: cyan). (B) Top: example fascicle connectivity profile in a single participant for a word-selective region, mOTS-words (black). Acronyms: pFus: posterior fusiform; mFus: mid fusiform; pOTS: posterior occipito-temporal sulcus. mOTS: mid occipito-temporal sulcus. IFOF: inferior fronto-occipital fasciculus; ILF: inferior longitudinal fasciculus; AF: arcuate fasciculus; pAF: posterior arcuate fasciculus; VOF; vertical occipital fasciculus. Bottom: Quantification of the connectivity profile shown above: the percentage of fibers connecting to these five fascicles. (C) Schematic of two opposing hypotheses relating the organization of white matter tracts to functional regions. Each panel shows the predicted distributions of connections across white matter fascicles for a given functional ROI. Colors: fascicle. The functional ROI arrangement mirrors the schematic in (A). Hypothesis 1: connectivity is linked to category. Hypothesis 2: connectivity is linked to cytoarchitecture.

We reasoned that if the white matter connections of ventral visual regions are linked to category-selectivity, then we would expect that (i) mFus-faces and pFus-faces would have similar white matter connections to support face recognition, (ii) mOTS-words and pOTS-words would have similar white matter connections to support word recognition, and (iii) white matter connections of word-selective regions would be different from those of face-selective regions (Fig 1C, Hypothesis 1). However, if the white matter connections of VTC are organized by cytoarchitecture, then we would expect (i) that mFus-faces and mOTS-words would have similar white matter connections as they reside 
in the same cytoarchitectonic area (FG4), (ii) that pFus-faces and pOTS-words would have similar white matter connections to each other (as they reside in FG2), and (iii) that the connectivity profile of regions in FG2 (pFus-faces and pOTS-worlds) will be different from those in FG4 (mFus-faces and mOTS-words) irrespective of category-selectivity (Fig 1C, Hypothesis 2). A third possibility is that white matter connections of VTC are linked to both cytoarchitecture and category-selectivity. This hypothesis predicts that each of the four functional ROls would have unique white matter connections.

In both children and adults, ventral face- and word-selective regions showed a nonuniform fascicle connectivity profile (Fig 2). Qualitatively, pFus-faces and pOTSwords showed prominent connections to the VOF, and mFus-faces and mOTS-words showed prominent connections to the pAF (example child in Fig 2A; all children in Supplemental Figs 1-4, all adults in Supplemental Figs 5-8).

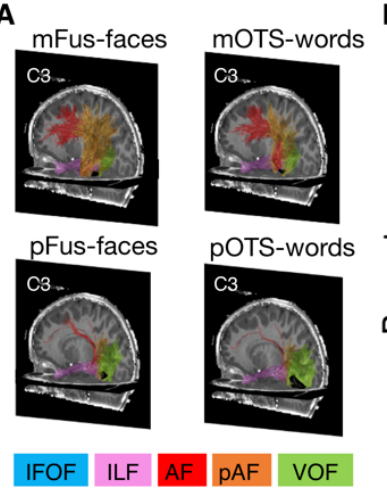

B

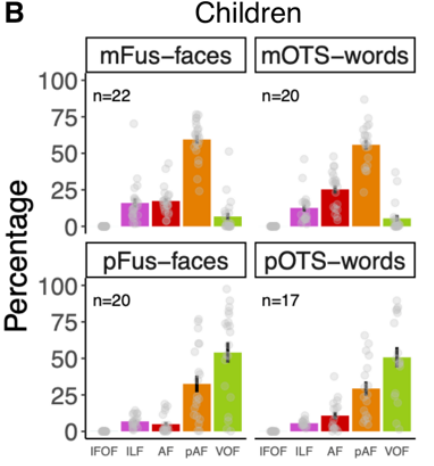

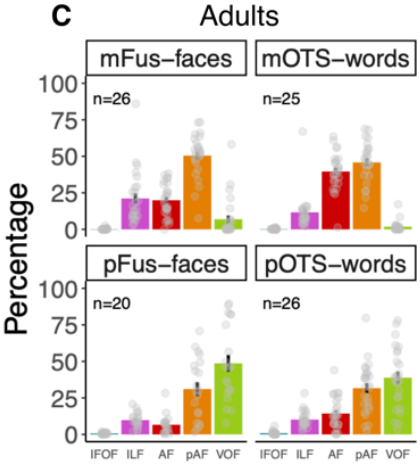

D

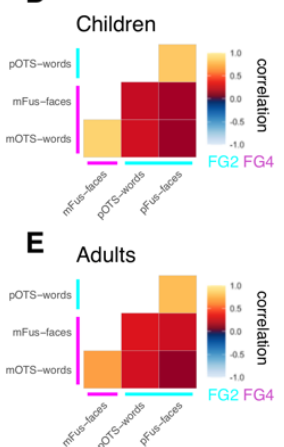

Figure 2. Fascicle connectivity profiles of VTC face- and word-selective regions. (A) Fascicle connectivity profiles of face- and word-selective functional ROIs in an example child (C3, 6-years-old). All subjects in Supplemental Figs 1-8. Acronyms: mFus-faces: mid fusiform face-selective region. pFusfaces: posterior fusiform face-selective region. mOTS-words: mid occipitotemporal sulcus word-selective region IFOF: inferior fronto-occipital fasciculus; ILF: inferior longitudinal fasciculus; $A F$ : arcuate fasciculus; $p A F$ : posterior arcuate fasciculus; VOF: vertical occipital fasciculus. (B,C) Histograms showing the percentage of connections to major fascicles for each of the functional regions in children $(B)$ and adults (C); Error bars: standard error of the mean; Dots: individual participant data. $(D, E)$ Correlation matrices depicting the average pairwise within-subject correlation between connectivity profiles for children (D) and adults (E). Lines depict cytoarchitectonic area; cyan: FG2, magenta: FG4.

In each participant, we quantified the fascicle connectivity profile for each functional ROI. That is, the percentage of fibers of each functional ROI that connect to each of these five fascicles (IFOF, ILF, AF, pAF, and VOF). Consistent with the qualitative observations, $\sim 50 \%$ of the white matter connections of mFus-faces and mOTS-words are to the pAF and $20-25 \%$ of the connections are to the ILF and AF, respectively (Fig 2B- 
top). Whereas $\sim 50 \%$ of the white matter connections of pFus-faces and pOTS-words are to VOF and $\sim 25 \%$ of the connections are to the pAF (Fig 2B-bottom). Similar fascicle connectivity profiles were observed in adults: $~ 50 \%$ of the white matter connections of mFus-faces and mOTS-words are to the pAF (Fig 2C-top) and $~ 40-50 \%$ connections of pFus-faces and pOTS-words are to the VOF (Fig 2C-bottom).

Fascicle connectivity profiles shown in Fig 2B,C suggest that the fascicle connectivity profiles are similar among functional ROls within the same cytoarchitectonic area consistent with the prediction that white matter is linked to cytoarchitecture (Fig 1Cright). To quantify the similarity between connectivity profiles, we calculated the correlation between fascicle connectivity profiles of face- and word-selective regions within and across categories and cytoarchitectonic areas. If fascicle connectivity profiles are linked to category-selectivity, then functional ROls selective for the same category would have connectivity profiles that are more correlated than functional ROls located within the same cytoarchitectonic area. However, if fascicle connectivity profiles are linked to cytoarchitecture, then functional ROls located within the same cytoarchitectonic area would have connectivity profiles that are more correlated than those selective for the same category.

The average within-subject lower triangular correlation matrix between fascicle connectivity profiles is shown in Fig 2D,E. Results demonstrate that the correlations between connectivity profiles of functional ROls within the same cytoarchitectonic area are higher than the correlations between functional ROls with the same categoryselectivity in both children (same cytoarchitectonic area: mOTS-words/mFus-faces correlation mean $\pm s d: 0.85 \pm 0.19$; pOTS-words/pFus-faces: $0.81 \pm 0.24$; same category: mFus-faces/pFus-faces: 0.15 \pm 0.42 ; mOTS-words/pOTS-words: $0.27 \pm 0.46$ ) and adults (same cytoarchitectonic area: mOTS-words/mFus-faces correlation mean \pm sd: 0.67 \pm 0.33 ; pOTS-words/pFus-faces: 0.77 \pm 0.30 ; same category: mFus-faces/pFus-faces: 0.26 \pm 0.49 ; mOTS-words/pOTS-words: 0.27 \pm 0.48 ). Similar results were observed in the right hemisphere (Supplemental Fig 9) and when controlling for motion and ROI size (Supplemental Fig 10).

The significance of factors was evaluated using analyses-of-variance with Satterthwaite approximations for degrees of freedom (Kuznetsova et al., 2017) (referred 
to as linear mixed model analysis of variance, LMM ANOVA). A 2-way repeatedmeasures LMM ANOVA on the correlation between fascicle connectivity profiles with factors of shared feature (cytoarchitecture/category-selectivity) and age group (children/adults) revealed a significant main effect of shared feature $(F(1,105.92)=75.07$, $p=5.65 \times 10^{-14}$ ) reflecting the overall higher correlations between connectivity profiles located within the same cytoarchitectonic area compared to those with the same category-selectivity. There was no significant main effect of age group $(F(1,44.56)=0.13$, $p=0.72$ ), suggesting that correlations between connectivity profiles of the various functional ROIs were not different across children and adults. Finally, there was no significant age group by shared feature interaction $(F(1,105.92)=2.19, p=0.14)$, reflecting that correlations were higher between connectivity profiles of functional ROls located within the same cytoarchitectonic area compared to functional ROls selective for the same category in both children and adults.

\section{Decoupling cytoarchitecture and distance}

One concern about these findings might be that functional ROls located within the same cytoarchitectonic area may be physically closer than functional ROls in different cytoarchitectonic areas. To test this possibility, we calculated the distances between functional ROls within and across cytoarchitectonic areas in each subject and tested if there were systematic differences in distances between functional ROls using a LMM repeated measures ANOVA with factors of cytoarchitecture area (same/different) and age group (children/adults). Functional ROls within the same cytoarchitectonic areas were on average $\sim 10 \mathrm{~mm}$ apart, but functional ROIs in different cytoarchitectonic areas were more than 15mm apart (Fig 3A-left). This difference was significant (main effect of cytoarchitecture: $\left.F(1,144)=91.44,<2 \times 10^{-16}\right)$, there was no significant effect of age group $(F(1,144)=0.71, p=0.40)$. However, there was a significant group by cytoarchitecture interaction $(F(1,144)=6.43, p=0.01)$. This suggests that ROls within the same cytoarchitectonic areas are closer than ROls in different cytoarchitectonic areas, but this difference is smaller in adults compared to children (Fig 3A- Functional ROI).

To assess if spatial proximity is driving the results, we conducted an additional analysis controlling for distance. To do so, we generated three equally spaced disk 
functional ROls on the fsaverage surface, transferred them to each participant's native cortical surface, and identified the functionally defined white matter for these control ROls

(Fig 3B). Two of these functional ROls were in FG4 (FG4-mid/FG4-ant) and one was in FG2 (FG2-mid) such that the middle functional ROI (FG4-mid) was located equally far from FG4-ant and FG2-mid (Fig 3A-control ROI). We reasoned that if distance drives the results, then the connectivity profile of FG4-mid would be equally correlated to that of FG4-ant and FG2-mid because they are equally far apart. However, if the correlation between FG4-mid and FG4-ant is significantly higher than between FG4-mid and FG2mid, then it would provide strong evidence that white matter connectivity profiles are coupled with cytoarchitecture, not distance.

A
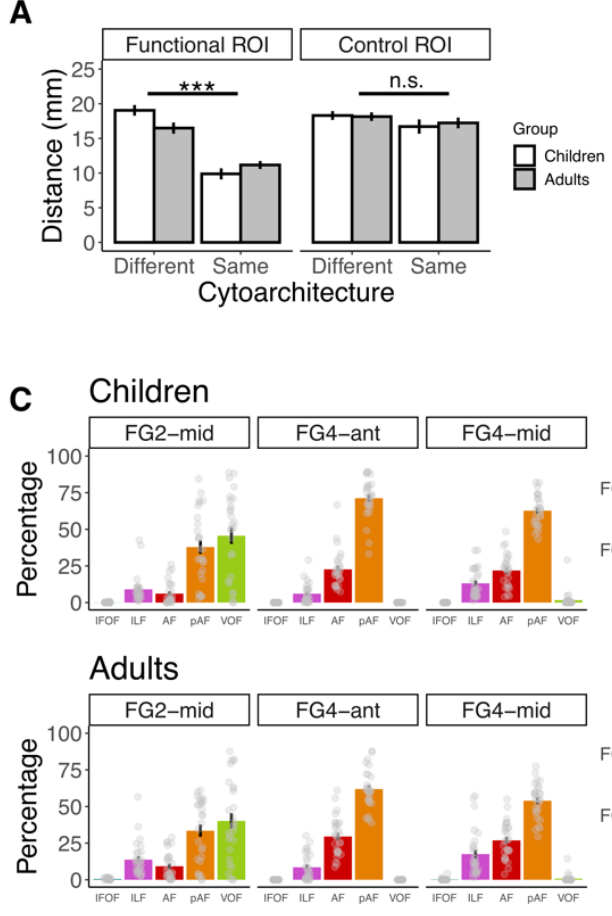

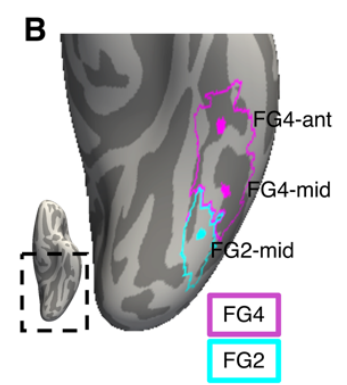

D
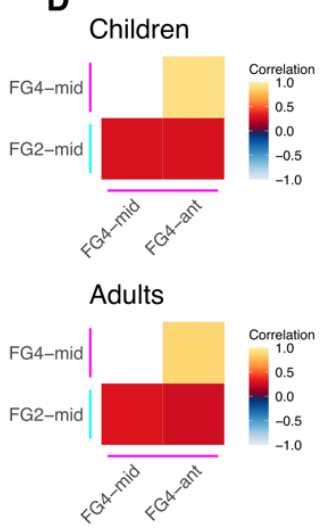

Figure 3. White matter is linked to cytoarchitecture when controlling for distance. (A) Average distance between functional ROls located within the same and different cytoarchitectonic area. Gray bars: children, white bars: adults. Error bars: standard error of the mean. Left: average distance between functional ROls, Right: average distance between control ROls. LMM ANOVA on distances of the control ROI revealed no main effect of cytoarchitecture $(F(1,106)=2.56$, $p=0.11)$, no main effect of age group $(F(1,106)=0.05, p=0.82)$ and no interaction $(F(1,106)=0.19$, $p=0.66))$. Significance: ${ }^{* * *} p<.001$. (B) Inflated fsaverage cortical surface depicting control ROls. Colors indicate cytoarchitectonic area: cyan: FG2, magenta: FG4. Acronyms: FG4-mid: control region in mid FG4. FG4-ant: control region in anterior FG4, FG2-mid: control region in mid FG2. Lines indicate cytoarchitectonic area: cyan: FG2; magenta: FG4. (C) Fascicle connectivity profiles for each of the equally spaced disk ROls in children (top) and adults (bottom); Error bars: standard error of the mean; Dots: individual participant data. Acronyms: IFOF: inferior fronto-occipital fasciculus; ILF: inferior longitudinal fasciculus; $A F$ : arcuate fasciculus; $p A F$ : posterior arcuate fasciculus; VOF: vertical occipital fasciculus. (D) Correlation matrices depicting the average within-subject correlation between fascicle connectivity profiles of equally spaced ROls in children (top) and adults (bottom).

Results reveal that in both children and adults, connectivity profiles (Fig 3C) of ROIs that are equally spaced apart and located in the same cytoarchitectonic area (FG4- 
mid and FG4-ant) are more similar than those across cytoarchitectonic areas (FG4-mid and FG2-mid). FG4-mid and FG4-ant are connected primarily to the pAF whereas FG2mid connects to both the VOF and pAF. Indeed, pairwise correlations between connectivity profiles are significantly higher among equally spaced ROls within the same cytoarchitectonic area than across cytoarchitectonic areas (Fig 4D, LMM ANOVA on the pairwise correlations between connectivity profiles with factors of same/different cytoarchitecture and age group reveal a significant main effect of cytoarchitecture $\left(F(1,53)=69.67, p=3.14 \times 10^{-11}\right.$, no significant differences across age groups $(F(1,53)=$ $0.07, p=0.79)$, and no group by cytoarchitecture interaction $(F(1,53)=0.26, p=0.61)$. These results indicate that connectivity profiles are linked to cytoarchitecture independent of the distance between the ROIs.

Do fascicle connectivity profiles of VTC ROIs predict cytoarchitecture or categoryselectivity?

We further hypothesized that if there is a consistent relationship between fascicle connectivity profile and cytoarchitecture or between fascicle connectivity profile and category, it should be possible to predict the cytoarchitectonic area or category-selectivity, respectively, from an unlabeled fascicle connectivity profile. We tested these hypotheses using a leave-one-out maximum correlation classification approach. That is, in each iteration, we calculated the average connectivity profile of each cytoarchitectonic area (FG2/FG4) and category (faces/words) using data from $n-1$ subjects. Then, we performed two separate two-way classifications of the left-out connectivity profiles based on the maximum correlation between the left-out connectivity profile and the average, to predict: (i) the cytoarchitectonic area (FG2 or FG4), and (ii) the category-selectivity (face or word).

For children (Fig 4A, light colors), the classifier successfully predicted the cytoarchitectonic area (mean classification accuracy $\pm s d=82 \% \pm 38 \% ; t(78)=7.47, p=$ $1.01 \times 10^{-10}$ ) above chance (50\%), but not category-selectivity (mean classification accuracy $\pm s d=49 \% \pm 50 \% ; t(78)=-0.11, p=0.91$ ). For adults (Fig 4A, dark colors), we successfully predicted both cytoarchitectonic area and category-selectivity above chance (cytoarchitecture: mean classification accuracy $\pm s d=87 \% \pm 34 \% ; t(96)=10.53, p=2.2 \times 10^{-}$ 16; category: mean classification accuracy $\pm s d=66 \% \pm 48 \% ; t(96)=3.31, p=0.001)$. A 2- 
way repeated-measures LMM ANOVA on classification accuracy with factors of classification feature (cytoarchitecture/category) and age group (children/adults) as factors revealed a significant main effect of classification feature $(F(1,348)=33.76, p=$ $1.41 \times 10^{-8}$ ) reflecting the overall higher classification accuracy of cytoarchitecture compared to category in both age groups. There was also a significant main effect of age group $(F(1,348)=5.16 p=0.02)$ reflecting higher classification accuracy for adult connectivity profiles than child connectivity profiles, and no significant interaction $(F(1,348)=1.78, p=0.18)$. Together, these analyses provide strong evidence supporting the hypothesis that fascicle connectivity profiles better predict cytoarchitecture rather than category-selectivity across age groups.

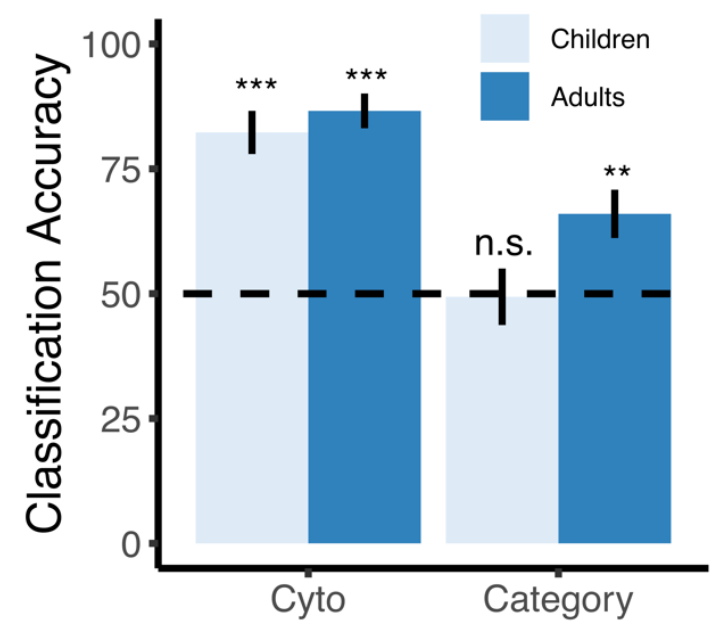

Figure 4: Fascicle connectivity profiles predict cytoarchitecture more than category. Average classification across participants of either the cytoarchitectonic area or category-selectivity from the fascicle connectivity profiles. Light blue: Children, dark blue: adults; Error bars: standard error of the mean. Dashed line: chance level. Significance vs. chance: ${ }^{*} p<.05,{ }^{* *} p<.01,{ }^{* * *} p$ $<$.001. n.s.: not significant.

Is there a unique fascicle connectivity profile for each functional region?

To test whether connectivity profiles are impacted by both cytoarchitecture and category-selectivity, we tested whether there is unique fascicle connectivity profile for each of the four VTC functional ROls. To do so, we performed a four-way classification to determine if it is possible to predict the functional ROI (mFus-faces, pFus-faces, mOTSwords, pOTS-words) from the fascicle connectivity profile. The average classification was significantly above chance $(25 \%)$ for both children (mean classification accuracy $\pm s d$ $=50 \% \pm 50 \% ; t(78)=4.53, p=2.11 \times 10^{-5}$ ) and adults (mean classification accuracy $\pm \mathrm{sd}=$ $\left.60 \% \pm 49 \% ; t(96)=6.95, p=4.34 \times 10^{-10}\right)$. Examination of classification accuracy by age group and functional ROI revealed that classification accuracy significantly varied by 
functional $\mathrm{ROI}(F(3,168)=4.17, p=0.007)$. Classification was not different between adults and children (Fig 5A, main effect of group $(F(1,168)=1.87, p=0.17)$, and there was no significant interaction $(F(3,168)=0.78, p=0.51)$.

A

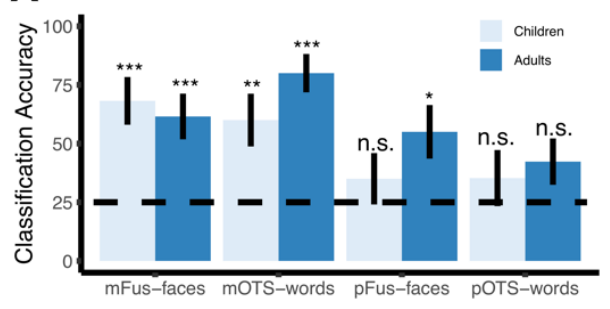

B

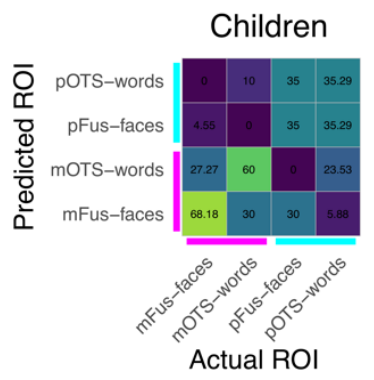

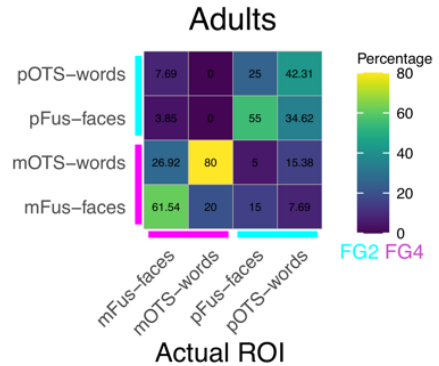

Actual ROI

Figure 5. Prediction of functional ROls from fascicle connectivity profiles (A) Average classification of each of the functional ROI across participants. Light blue: Children, dark blue: adults; Error bars: standard error of the mean. Dashed line: chance level. Significantly different than chance: ${ }^{*} p<.05,{ }^{* *} p<.01,{ }^{* * *} p$ $<.001$. n.s.: not significant. (B) Confusion matrix for the average classification of the functional ROI across participants. Each cell depicts the percentage of times each actual functional ROI (x-axis) was predicted (y-axis), see colorbar. On-diagonals: \% successful classification; off-diagonals: \% miss-classification. Each column sums to $100 \%$. Acronyms: mFus-faces: mid fusiform face-selective region. $p$ Fus-faces: posterior fusiform face-selective region. mOTS-words: mid occipitotemporal sulcus word-selective region. $p O T S-$ words: posterior occipitotemporal sulcus word-selective region. Cytoarchitectonic areas: cyan: FG2, magenta: FG4.

The classification confusion matrix enables us to examine whether misclassifications occurred more often between functional ROls located in the same cytoarchitectonic area or between functional ROls with the same category preference. Results reveal that in both children and adults functional ROls were more often confused with the other functional ROI in the same cytoarchitectonic area (Fig 5B). Indeed, a linear model on the percentage of times each functional ROI was misclassified as another functional ROI with factors of cytoarchitectonic area (same/different), category (same/different), and group (children/adults) revealed a significant main effect of cytoarchitectonic area $\left(F(1,18)=52.67, p=9.54 \times 10^{-7}\right)$ and a smaller but significant main effect of category $(F(1,18)=7.92, p=0.01$, all other effects and interactions were not significant; $F s<3.2 p s>0.09$ ).

Overall, the analyses of the fascicle connectivity profiles of ventral functional ROls suggest that there is a tight link between fascicle connectivity profiles and cytoarchitecture. Fascicle connectivity profiles predict cytoarchitecture better than 
category-selectivity and errors in predicting functional ROI from connectivity profiles occur more often for functional ROls located within the same cytoarchitectonic area.

Endpoint connectivity profiles of VTC functional ROIs are organized by cytoarchitecture

While the fascicle connectivity profile reveals which fascicles connect to each functional ROI, it is also important to understand the endpoint connectivity profiles of the white matter connections of each functional ROI. The endpoint connectivity profile provides complementary data to the fascicle connectivity profile for three reasons: (i) Not all fibers in the whole brain connectome are part of major fascicles; e.g., many fibers connecting VTC to early visual cortex are not classified as part of the major fascicles in common atlases (Catani et al., 2002; Wakana et al., 2004). (ii) Endpoint profiles provide additional information as to which cortical regions (Glasser et al., 2016) VTC functional ROls connect to, and (iii) functional ROls may share fascicles but have different endpoints, e.g., mOTS and mFus may connect to the arcuate fasciculus with only mOTS having endpoints in Broca's area.

Therefore, we next examined the endpoint connectivity profiles of the VTC functional ROls. To do so, we intersected each of the face- and word-selective functional ROls with the whole brain connectome of each participant. To determine the cortical regions to which these white matter tracts connect, we projected the tract endpoints to the cortical surface, and then used the Glasser Atlas (Glasser et al., 2016) to quantify the distribution of the endpoints across the brain.

Fig 6 shows the average spatial distribution across cortex of white matter endpoints for each of the VTC face and word-selective functional ROls in children (Fig 6A) and adults (Fig 6B). Qualitatively, the spatial distribution of endpoints is similar between anterior functional ROls (mFus-faces and mOTS-words) as well as similar between posterior functional ROIs (pFus-faces and mOTS-words. The anterior functional ROIs (mFus-faces and mOTS-words) show a distinct spatial distribution of endpoints with the highest endpoint density in ventral occipital and anterior temporal cortex, followed by endpoints in parietal cortex and inferior frontal cortex. Indeed, for mFus-faces and mOTSwords, the Glasser ROls with the highest mean endpoint proportions were in ventral temporal cortex (Children top five Glasser ROls: mFus-faces: FFC, VVC, PHA3, TE2p, 
and PH; mOTS-words: FFC, PH, TE2p, VVC, and PHA3. Adults top 5: mFus-faces: FFC, VVC, PHA3, V8, VMV3; mOTS-words: PH, FFC, TE2p, VVC, and one lateral temporal: FST).
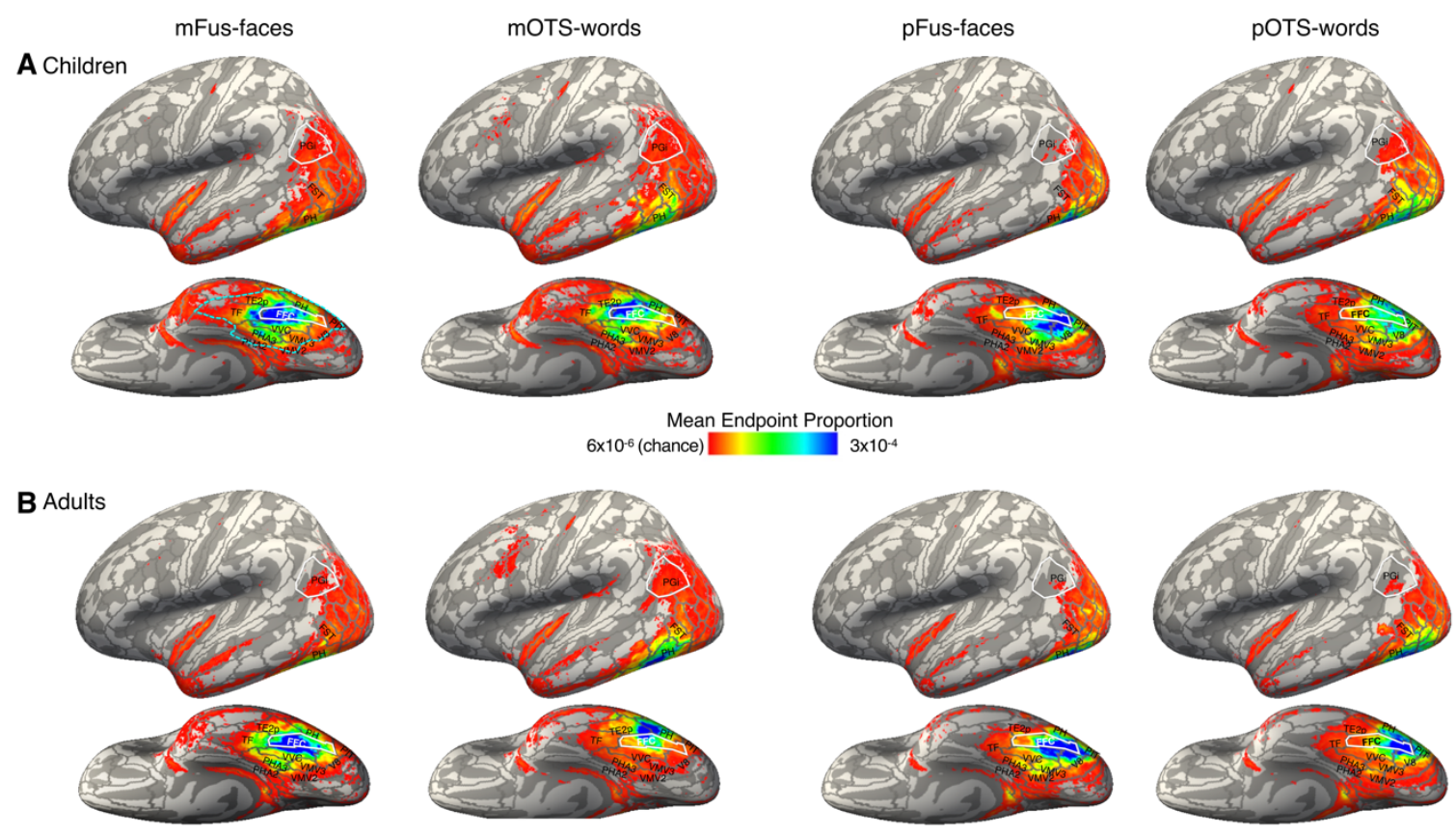

Figure 6. Endpoint connectivity profile of face- and word-selective regions across the entire brain. Average endpoint connectivity profile of mFus-face, pFus-faces, mOTS-words, pOTS-words in children (A) and adults (B). Data are shown on the fsaverage inflated cortical surface. Color map: endpoint proportion at each vertex (each map sums to one). Threshold value of maps: chance level if all endpoints were evenly distributed across the brain. Gray outlines: Glasser ROls. Cyan outline: ventral temporal cortex. White outlines: PGi and FFC as a visual reference to aid comparison across panels.

In contrast, the posterior functional ROIs (pFus-faces and pOTS-words) illustrate the highest endpoint density in ventral temporal and occipital cortex, followed by the posterior parietal cortex and anterior temporal cortex. For pFus-faces, the top five Glasser ROls were in ventral temporal (Children: V8, FFC, VVC, PIT, VMV3; Adults: V8, FFC, PIT, VVC, VMV3). For pOTS-words, four of the top five Glasser ROIs were ventral (Children: V8, PIT, PH, FFC; adults: PIT, FFC, V8, PH) and one was lateral-occipital (Children \& Adults: LO2). In other words, in the occipital and parietal cortices, the endpoint density for posterior face- and word-selective regions appears to be shifted more posteriorly compared to that of anterior face- and word-selective regions.

We next quantified the endpoint distributions of each of the functional ROls across the brain using the Glasser Atlas parcellation in children (Fig 7A) and adults (Fig 7B). 
Right hemisphere data is in Supplemental Fig 11. Consistent with the qualitative analysis, the endpoint distributions for ROls located in the same cytoarchitectonic area are similar. That is, pFus-faces and pOTS-words have similar endpoint distributions, with the highest endpoint densities within the occipital lobe, and mFus-faces and mOTS-words have similar endpoint distributions, with higher endpoint proportions in the temporal and occipital lobes than the parietal and frontal lobes. Interestingly, mFus-faces and mOTSwords appear to have more distinct endpoint distributions in adults (Fig 7B) compared to children (Fig 7A), especially in the occipital lobe.

A

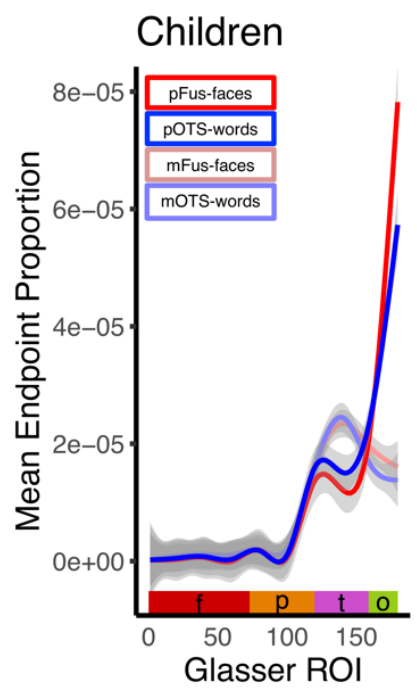

B

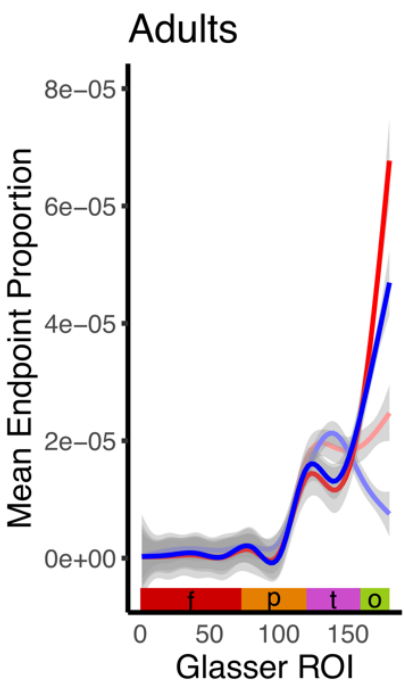

C

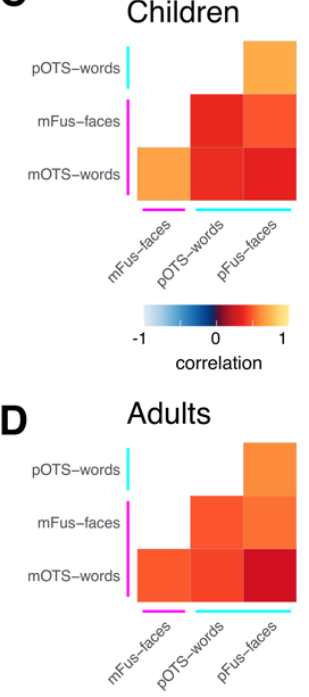

E

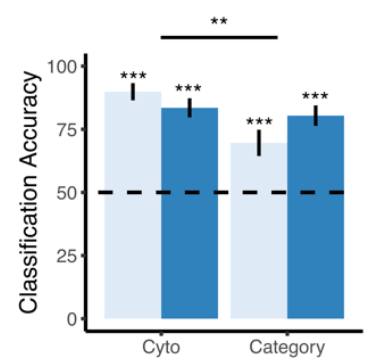

Group

Children

Adults

Figure 7. Quantification of endpoint connectivity profile of face- and word-selective regions in children and adults. (A,B) Mean endpoint proportion across 180 Glasser ROls for each of the VTC word and face-selective regions in children (A) and adults (B). Line color indicates the functional ROI: red: $p F u s-$ faces; blue: pOTS-words; light red: mFus-faces; light blue: mOTS-words. Shaded area: standard error of the mean. $X$-axis: Glasser Atlas ROI number arranged by lobe: red: frontal (f); orange: parietal (p); magenta: temporal (t); green: occipital (o). (C,D) Lower triangular correlation matrices depicting the average withinsubject pairwise correlation between endpoint connectivity profiles of ventral face and word-selective ROls in children (C) and adults (D). (E) Bar graphs showing classification accuracy of either the cytoarchitectonic area or category-selectivity from the endpoint connectivity profiles. Light blue: Children, dark blue: adults; Error bars: standard error of the mean. Dashed line: chance level. Significantly different than chance: ${ }^{*} p<$ $.05,{ }^{* *} p<.01,{ }^{* * *} p<.001$. n.s.: not significant. Acronyms: mFus-faces: mid fusiform face-selective region. pFus-faces: posterior fusiform face-selective region. mOTS-words: mid occipitotemporal sulcus wordselective region. pOTS-words: posterior occipitotemporal sulcus word-selective region.

To quantitatively test our hypotheses (Fig 1B), we calculated the correlation between the endpoint density profiles of all pairs of functional ROIs, and then tested if correlations between endpoint profiles were higher among functional ROls within the 
same cytoarchitectonic area or among functional ROls selective for the same category. The lower triangular correlation matrix in Fig 7C-left demonstrates that for children, functional ROls within the same cytoarchitectonic area had higher correlations among endpoint profiles compared to functional ROls selective for the same category (mean correlation $\pm s d$, same cytoarchitectonic area: mOTS-words/mFus-faces: 0.68 \pm 0.24 ; pOTS-words/pFus-faces: 0.71 \pm 0.20 ; same category: mFus-faces/pFus-faces: 0.48 \pm 0.19 ; mOTS-words/pOTS-words: 0.38 \pm 0.16 ). For adults (Fig 7D-right), however, this pattern was less clear (same cytoarchitectonic area: mFus-faces/mOTS-words $0.49 \pm 0.24$, pFusfaces/pOTS-words 0.60 \pm 0.24 ; same category: mFus-faces/pFus-faces: $0.54 \pm 0.22$, mOTS-words/pOTS-words: 0.44士0.21). A 2-way repeated-measures LMM ANOVA on correlations between connectivity profiles with factors of shared feature (cytoarchitecture/category-selectivity) and age group (children/adults) revealed a significant main effect of shared feature $\left(F(1,104.13)=22.12, p=7.91 \times 10^{-6}\right)$ reflecting the overall higher correlations between connectivity profiles of functional ROls located within the same cytoarchitectonic area compared to selective for the same category. However, there was also a significant shared feature by age group interaction $(F(1,104.13)=8.91, p=0.004 ;$ no significant effect of age group $(F(1,45.38)=1.46, p=$ 0.23), reflecting that in children, but not adults, connectivity profiles were more correlated between functional ROIs located within the same cytoarchitectonic area compared to those selective for the same category (children: $t(58.041)=5.14, p=3.14 \times 10^{-6}$; adults: $t(81.09)=1.14, p=0.26)$. In other words, we find evidence for significant developmental differences in endpoint connectivity profiles. In children, but not adults, the endpoint connectivity profiles are more correlated among functional ROls within the same cytoarchitectonic area compared to functional ROls selective for the same category.

As we found an age by feature interaction and prior literature suggests that the spatial extent of face and word-selective functional ROls increases from childhood to adulthood (Golarai et al., 2010), we asked if these age group differences were due to developmental changes to the functional ROls. Thus, we repeated endpoint profile analyses in two ways. First, to control for functional ROI size, we repeated these analyses using constant size disk ROls placed at the center of each participant's functional ROI (Supplemental Fig 12B). Second, to test if these developmental effects may be driven 
by the high endpoint density in VTC Glasser ROls adjacent to the functional ROls, we repeated the endpoint analyses using all Glasser ROls except 11 Glasser ROIs that are within VTC and overlap the category-selective regions (see methods, Supplemental Fig 12C). We reasoned that if group differences will be eliminated under these controls it would suggest that developmental changes in VTC are driving age group differences in connectivity profiles. Alternatively, finding a similar pattern of results in the control analyses would provide strong evidence for development differences in endpoint connectivity profiles that supersede local changes in the VTC. Both control analyses replicate the findings in Fig 7. That is, in all analyses we find significantly higher correlations among endpoint connectivity profiles of functional ROls within the same cytoarchitectonic areas than among functional ROls of the same category preference and significantly more so in children than adults (Supplemental Fig 12A,C,E).

To further examine the predictive nature of the endpoint connectivity profiles, we used a leave-one-out maximum correlation classification approach to test whether we can predict cytoarchitectonic area (FG2/FG4) or category-selectivity (faces/words) from an unlabeled endpoint connectivity profile. We could successfully predict cytoarchitecture above chance for both children (Fig 7E-light colors, mean classification accuracy \pm sd: $90 \% \pm 30 \% ; t(78)=11.67, p=2.2 \times 10^{-16}$ ) and adults (Fig 7E-dark colors, mean accuracy \pm sd: $\left.84 \% \pm 37 \% ; t(96)=8.84, p=4.49 \times 10^{-14}\right)$. We could also predict categoryselectivity above chance for children (mean accuracy \pm sd: $70 \% \pm 46 \% ; t(78)=3.77, p=$ 0.0003 ) and adults (mean accuracy \pm sd: $80 \% \pm 40 \% ; t(96)=7.51, p=3.07 \times 10^{-11}$ ). Overall, classification of cytoarchitectonic area was significantly higher than category-selectivity (main effect of classification type: $F(1,298.11)=7.97, p=0.005$, 2-way repeatedmeasures LMM ANOVA on classification accuracy with factors of classification feature (cytoarchitecture/category-selectivity) and age group (children/adults)). However, there was a significant difference across age groups (significant classification type by group interaction $(F(1,298.11)=4.31, p=0.03$; no main effect of group $F(1,49.01)=0.28, p=$ $0.60)$ : In children, classification of cytoarchitecture was higher than that of categoryselectivity (paired t-test: $t(78)=3.20, p=0.002$ ) but in adults, this was not the case (paired t-test: $t(96)=0.60, p=0.55)$. Results were largely similar when controlling for functional ROI size and excluding Glasser ROIs in VTC (Supplemental Fig 12B,D,F). These results 
suggest that in childhood, endpoint connectivity profiles predict cytoarchitecture better than category-selectivity, but by adulthood, this is no longer the case.

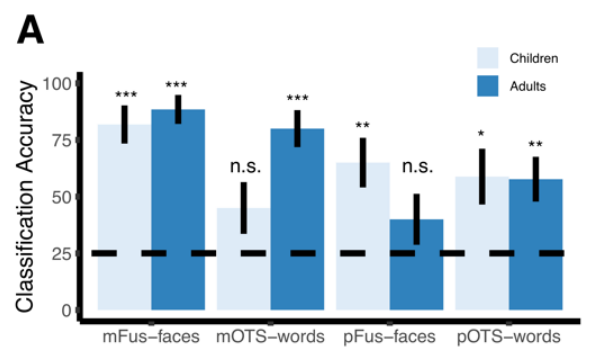

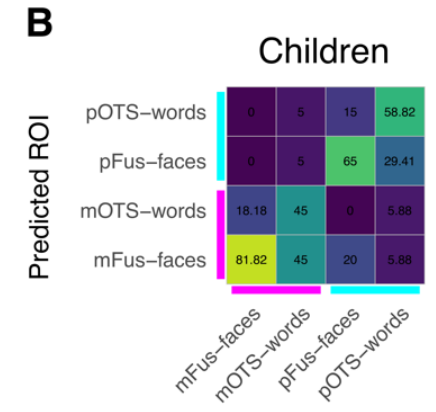

Actual ROI

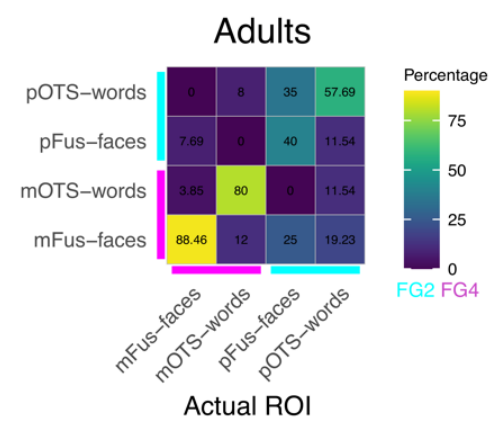

Figure 8. Prediction of functional ROls from endpoint connectivity profiles. (A) Average classification of each of the functional ROI across participants. Light blue: Children, dark blue: adults; Error bars: standard error of the mean. Dashed line: chance level. Significantly different than chance: ${ }^{*} p<.05,{ }^{* *} p<.01,{ }^{* * *} p$ $<$.001. n.s.: not significant. (B) Average classification of the functional ROI across participants. Each cell depicts the percentage of times each actual functional ROI (x-axis) was predicted (y-axis), see colorbar. Each column sums to $100 \%$. On-diagonals: \% successful classification; off-diagonals: \% misclassification. Acronyms: mFus-faces: mid fusiform face-selective region. $p F u s-f a c e s:$ posterior fusiform face-selective region. mOTS-words: mid occipitotemporal sulcus word-selective region. pOTS-words: posterior occipitotemporal sulcus word-selective region. Cytoarchitectonic areas: cyan: FG2, magenta: FG4.

Lastly, we asked, does development lead to a unique endpoint connectivity profile for each of the four functional ROls in adults? We examined this possibility by testing if the functional ROI (mFus-faces, pFus-faces, mOTS-words, pOTS-words) can be predicted from an unlabeled left-out endpoint connectivity profile, and if this classification is higher in adults than children. The average four-way functional ROI classification was significantly above $25 \%$ chance for both children (mean classification accuracy \pm sd: $63 \% \pm 48 \% ; t(78)=7.02, p=7.35 \times 10^{-10}$ ) and adults (mean classification accuracy $\pm s d$ : $\left.68 \% \pm 47 \% ; t(96)=9.05, p<1.69 \times 10^{-14}\right)$. A LMM ANOVA on the four-way functional ROI classification with factors of functional ROI (mFus-faces, mOTS-words, pFus-faces, pOTS-words) and age group (children/adults) revealed a significant effect of functional $\mathrm{ROI}(F(3,132.80)=4.49, p=0.005)$, a significant functional $\mathrm{ROI}$ by group interaction $(F(3,132.80)=3.18, p=0.03)$, but no significant effect of age group $(F(1,50.88)=0.31, p$ $=0.58)$. This indicates a differential classification of functional ROI from endpoint connectivity across age groups. Anterior functional ROIs (mFus-faces and mOTS-words) were better classified in adults than children, but the opposite was true for posterior functional ROls (pFus-faces and pOTS-words, Fig 8A). Interestingly, the largest 
developmental increase in accuracy was observed in mOTS-words, as classification of mOTS-words from endpoint connectivity profiles almost doubled from children to adults. Finally, examination of the classification confusion matrix revealed that functional ROls were significantly more often confused with other functional ROls in the same cytoarchitectonic area (Fig 8B, main effect of cytoarchitectonic area $(F(1,18)=10.57, p$ $=.004$, linear model on the percentage of times each functional ROI was misclassified as each other functional ROI with factors of cytoarchitectonic area (same/different), category (same/different), and group (children/adults); no other main or interaction effects were significant, Fs<2.97; ps>0.10).

Overall, the endpoint connectivity profile analysis of ventral functional ROls suggests that initially in childhood there is a tight link between endpoint connectivity profiles and cytoarchitecture, as endpoint connectivity predicts cytoarchitecture better than category-selectivity and confusability is largely among functional ROls within the same cytoarchitectonic area. However, development from childhood to adulthood in the endpoint connectivity of anterior functional ROIs, and particularly mOTS-words, results in more distinct endpoint connectivity profiles for the functional ROls within FG4.

\section{Discussion}

Here, we tested whether white matter connections of category-selective functional ROIs in the ventral stream are linked to category-selectivity or cytoarchitecture. We find that in both children and adults, white matter connectivity better predicts cytoarchitecture than category-selectivity. In adulthood, however, connectivity profiles for functional ROIs located within the same cytoarchitectonic region became more distinct, particularly in FG4. These results suggest that in childhood, cytoarchitecture and white matter largely co-vary together. However, over development connectivity profiles may become more specialized to support category-specific processing.

Rethinking the relationship between white matter and category-selectivity 
Past work has suggested that category-selective regions have specific white matter tracts to support category-selective processing (Bouhali et al., 2014; Mahon \& Caramazza, 2011). Some of the most compelling work demonstrating the relationship between category-selectivity and white matter connectivity are fingerprinting studies that have shown that it is possible to predict category-selective responses from the endpoint connectivity profile across the brain (Grotheer, Yeatman, et al., 2021; Osher et al., 2016; Saygin et al., 2011), and further that it is possible to predict from endpoint connectivity at age five where word-selective activations will emerge in VTC at age eight (Saygin et al., 2016). In this study, Saygin et al., (2016) found that the correlation between the predicted word-selectivity and actual word selectivity from the white matter fingerprint is $0.47 \pm$ 0.036 (mean across subjects, Fisher $\mathrm{z}$ ). It is interesting that we find that the correlation between connectivity profiles of functional ROls selective for the same category is comparable (children: $0.49 \pm 0.04$, adults: 0.59 \pm 0.05 , mean Fisher z). Strikingly, however, the correlation between connectivity profiles for functional ROls located in the same cytoarchitectonic area is much higher (children:1.03 \pm 0.11 , adults:0.69 \pm 0.06 , mean Fisher z). We also predict category-selectivity from white matter connectivity above chance for both children and adults. However, by testing predictivity of white matter for multiple attributes including ones that have not been tested before, we find that connectivity profiles predict cytoarchitecture significantly better than category-selectivity.

It is important to note that some category-selective regions that were tested in prior studies are also located in different cytoarchitectonic areas. For example, face-selective and place-selective regions have both unique cytoarchitecture (Weiner, Barnett, et al., 2017) and unique white matter connectivity (Gomez et al., 2015; Saygin et al., 2011). Therefore, comparing the white matter connectivity profiles of face- and place-selective regions may lead to the inaccurate conclusion that category-selectivity, cytoarchitecture, and white-matter are all linked. Instead, our study shows that this is not the general case, and it is necessary to examine cases where category-selective and cytoarchitecture are orthogonal to reveal the broader principle that white matter is linked to cytoarchitecture above category-selectivity.

Indeed, the results of the present study generate new predictions about other category-selective regions beyond word- and face-selective regions in VTC. Prior data 
shows that place-selective CoS-places is located in cytoarchitectonic area FG3 (Weiner et al., 2017) and body-selective OTS-bodies is located in cytoarchitectonic area FG4 (Weiner et al., 2017). Therefore, our findings predict that OTS-bodies would have a similar connectivity profile to mFus-faces and mOTS-words, which are also located in FG4, and CoS-places would have a distinct connectivity profile from all the other functional ROls examined in our study. To test these predictions, we compared the fascicle connectivity profiles of OTS-bodies and CoS-places to the connectivity profiles of other functional ROls and we find that this is indeed the case (Fig 9).
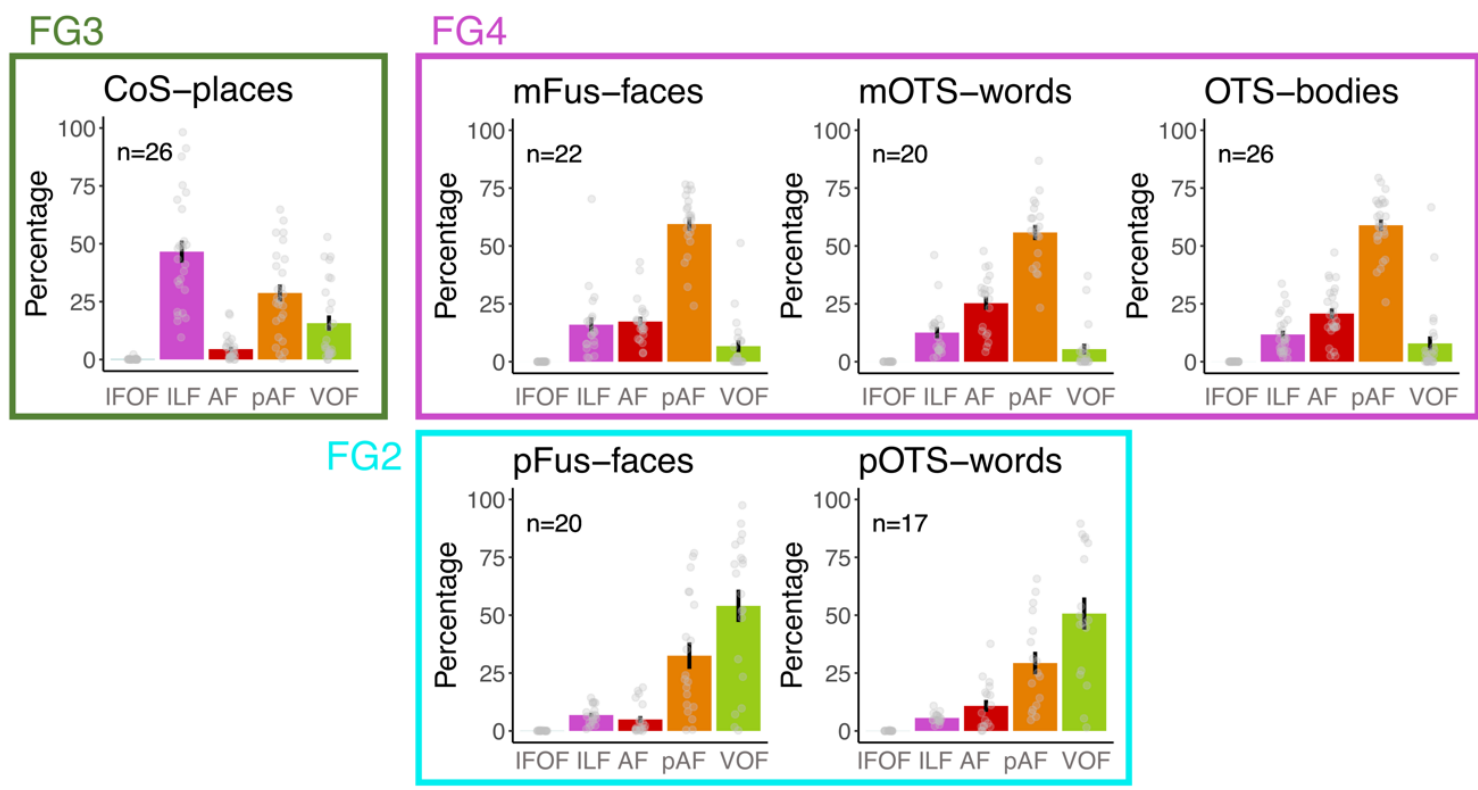

Figure 9. Fascicle connectivity profiles of additional VTC category-selective regions. Histograms showing the percentage of connections to major fascicles for each of the functional regions in children. Error bars: standard error of the mean; Dots: individual participant data. Acronyms: CoS-places: collateral sulcus place-selective region. mFus-faces: mid fusiform face-selective region. mOTS-words: mid occipitotemporal sulcus word-selective region. OTS-bodies: occipitotemporal sulcus body-selective region. pFus-faces: posterior fusiform face-selective region. pOTS-words: posterior occipitotemporal sulcus wordselective region. IFOF: inferior fronto-occipital fasciculus; ILF: inferior longitudinal fasciculus; $A F$ : arcuate fasciculus; $p A F$ : posterior arcuate fasciculus; VOF: vertical occipital fasciculus. Boxes depict cytoarchitectonic area, cyan: FG2, green: FG3, magenta: FG4.

\section{Developmental Implications}

The theory that white matter is linked to category-selectivity also has developmental implications. For example, it has been proposed that white matter early in childhood provides innate scaffolding for category-selectivity and that innate white matter connections might be a mechanism which explains similarities in the functional 
organization of visual cortex between blind and sighted participants (Bi et al., 2016; Mahon \& Caramazza, 2011; Murty et al., 2020; van den Hurk et al., 2017).

Here, we find that early in childhood white matter is better tied to cytoarchitecture than category, but over development category-specific connections emerge. These findings suggest a rethinking of the view that white matter tracts provide an innate scaffold for category-selective function. Instead, it seems that white matter and cytoarchitecture are closely linked early in development, and that category-specific connections can be tuned over development. Recent findings revealing that white matter fascicles can be reliably identified in individual newborns (Grotheer, Rosenke, et al., 2021) pave the way for future research to examine whether the relationship between cytoarchitecture and white matter connections is present at birth.

Another interesting direction for future research is understanding the mechanisms that enable tuning of white matter connections during childhood development. One possibility is that white matter connections may grow or prune to support the more specific connections needed for brain networks to better support specific behaviors. For example, learning to read may require fine-tuning of connections between visual areas and language areas in the prefrontal cortex. Alternatively, as functional regions in VTC develop and grow, they may shift to the edges of cytoarchitectonic areas, thus forming unique connections that span multiple networks. Since white matter connections are domain general early in development, one implication from this study is that interventions targeting certain skills (e.g., reading) may be more successful before white matter connections gain category-specificity.

\section{What is the utility of shared connectivity?}

While past work has argued for the utility of specific white matter connections to support domain-specific processing, there are also reasons why it might be useful to have white matter connections that are domain-general. Similar white matter connections of functional regions within a cytoarchitectonic area suggest that regions within the same cytoarchitectonic may perform similar computations. An example of such a shared computation across the domains of reading and face recognition is the processing of 
foveal information which requires high visual acuity (Hasson et al., 2002; Levy et al., 2001).

An additional benefit of shared white matter connections for functional regions selective for different categories may be flexibility. A large body of research has revealed that learning can lead to the development of new category-selectivity in VTC. For example, illiterate children and adults do not have word-selective regions in VTC; however, both groups develop word-selectivity after learning to read (Brem et al., 2010; Dehaene et al., 2010; Saygin et al., 2016). A flexible and domain-general system may enable new selectivity to emerge as it becomes relevant, as well as older selectivity (e.g., selectivity to limbs) to be recycled (Nordt et al., 2021). Since it is not possible to predict which categories will be behaviorally relevant (e.g., children can learn artificial categories such as Pokémon (Gomez et al., 2019)), a domain-general architecture provides flexibility to develop many category representations.

In addition to shared computational demands for regions selective for different categories, regions in different cytoarchitectonic areas may be performing different computations on the same stimulus. For example, classic studies suggested that unique cytoarchitecture and white matter enable parallel processing of different attributes of a stimulus (such as its motion and color) (Van Essen et al., 1992; Zeki \& Shipp, 1988). In the context of VTC, prior research has demonstrate that word-selective regions located within different cytoarchitectonic regions perform different computations on the same stimuli (Caffarra et al., 2021; Lerma-Usabiaga et al., 2018; White et al., 2019). These studies have shown that (i) pOTS-words can process two words in parallel, whereas mOTS-words can only process one word at a time (White et al., 2019) and (ii) pOTSwords is involved in extracting visual features, whereas mOTS-words is involved in processing lexical features (Lerma-Usabiaga et al., 2018). Therefore, we propose that multiple regions that are selective for the same category but located in different cytoarchitectonic regions and have different white matter connections may perform different computations towards a shared visual behavior.

Beyond VTC 
In addition to elucidating the pattern of white matter connections of the human visual system, this work has broader implications for the rest of the brain. Specifically, we hypothesize that across the brain, there are tight links between white matter connections and cytoarchitecture. Other evidence supporting this idea comes from past work showing that supplementary motor area (SMA) and pre-SMA which are in distinct cytoarchitectonic regions also have distinct connectivity profiles (Johansen-Berg et al., 2004; Klein et al., 2007), as well as studies that showed that Broca's area spans multiple cytoarchitectonic regions (Amunts et al., 2010) with distinct connectivity profiles (Klein et al., 2007). Future research can examine this hypothesis in other sensory-motor (Heil et al., 2005; Morosan et al., 2005; Shipp, 2003) and cognitive systems (Siegel et al., 2012; Sporns et al., 2005) across the brain.

\section{Summary}

In school-aged children and adults the white matter connections of ventral functional ROls are linked to cytoarchitecture. Over development, white matter connectivity profiles become more distinct for functional ROls with different functional selectivity located within the same cytoarchitectonic region. These findings suggest that cytoarchitecture and white matter are linked early in childhood and become more specific during development. Together our data propose a rethinking of the view that categoryselective regions have innate and specific white matter connections to support categoryspecific processing.

Acknowledgments: This work was supported by an NSF Graduate Research Fellowship (DGE-1656518) awarded to EK and a NIH grant (RO1 EY 022318-06) awarded to KGS. We thank Brianna Jeska and Michael Barnett for their contributions in scanning participants. 


\section{References}

Amunts, K., Lenzen, M., Friederici, A. D., Schleicher, A., Morosan, P., Palomero-Gallagher, N., \& Zilles, K. (2010). Broca's region: Novel organizational principles and multiple receptor mapping. PLoS Biology, 8(9). https://doi.org/10.1371/journal.pbio.1000489

Amunts, K., \& Zilles, K. (2015). Architectonic Mapping of the Human Brain beyond Brodmann. Neuron, 88(6), 1086-1107. https://doi.org/10.1016/j.neuron.2015.12.001

Ben-Shachar, M., Dougherty, R. F., \& Wandell, B. A. (2007). White matter pathways in reading. Current Opinion in Neurobiology, 17(2), 258-270.

https://doi.org/10.1016/j.conb.2007.03.006

Bi, Y., Wang, X., \& Caramazza, A. (2016). Object Domain and Modality in the Ventral Visual Pathway. Trends in Cognitive Sciences, 20(4), 282-290.

https://doi.org/10.1016/j.tics.2016.02.002

Bouhali, F., Thiebaut de Schotten, M., Pinel, P., Poupon, C., Mangin, J.-F., Dehaene, S., \& Cohen, L. (2014). Anatomical Connections of the Visual Word Form Area. Journal of Neuroscience, 34(46), 15402-15414. https://doi.org/10.1523/JNEUROSCI.4918-13.2014

Brem, S., Bach, S., Kucian, K., Kujala, J. V., Guttorm, T. K., Martin, E., Lyytinen, H., Brandeis, D., \& Richardson, U. (2010). Brain sensitivity to print emerges when children learn letterspeech sound correspondences. Proceedings of the National Academy of Sciences, 107(17), 7939-7944. https://doi.org/10.1073/pnas.0904402107

Broce, I. J., Bernal, B., Altman, N., Bradley, C., Baez, N., Cabrera, L., Hernandez, G., De Feria, A., \& Dick, A. S. (2019). Fiber pathways supporting early literacy development in 5-8-year- 
old children. Brain and Cognition, 134, 80-89.

https://doi.org/10.1016/j.bandc.2018.12.004

Butt, O. H., Benson, N. C., Datta, R., \& Aguirre, G. K. (2013). The Fine-Scale Functional Correlation of Striate Cortex in Sighted and Blind People. Journal of Neuroscience, 33(41), 16209-16219. https://doi.org/10.1523/JNEUROSCI.0363-13.2013

Caffarra, S., Karipidis, I. I., Yablonski, M., \& Yeatman, J. D. (2021). Anatomy and physiology of word-selective visual cortex: From visual features to lexical processing. Brain Structure \& Function, 226(9), 3051-3065. https://doi.org/10.1007/s00429-021-02384-8

Catani, M., Howard, R. J., Pajevic, S., \& Jones, D. K. (2002). Virtual in Vivo Interactive Dissection of White Matter Fasciculi in the Human Brain. Neurolmage, 17(1), 77-94. https://doi.org/10.1006/nimg.2002.1136

Cohen, L., Martinaud, O., Lemer, C., Lehéricy, S., Samson, Y., Obadia, M., Slachevsky, A., \& Dehaene, S. (2003). Visual word recognition in the left and right hemispheres: Anatomical and functional correlates of peripheral alexias. Cerebral Cortex (New York, N.Y.: 1991), 13(12), 1313-1333. https://doi.org/10.1093/cercor/bhg079

Deen, B., Richardson, H., Dilks, D. D., Takahashi, A., Keil, B., Wald, L. L., Kanwisher, N., \& Saxe, R. (2017). Organization of high-level visual cortex in human infants. Nature Communications, 8(1), 13995. https://doi.org/10.1038/ncomms13995

Dehaene, S., \& Cohen, L. (2007). Cultural Recycling of Cortical Maps. Neuron, 56(2), 384-398. https://doi.org/10.1016/j.neuron.2007.10.004

Dehaene, S., Pegado, F., Braga, L. W., Ventura, P., Filho, G. N., Jobert, A., Dehaene-Lambertz, G., Kolinsky, R., Morais, J., \& Cohen, L. (2010). How Learning to Read Changes the Cortical 
Networks for Vision and Language. Science, 330(6009), 1359-1364.

https://doi.org/10.1126/science.1194140

Dehaene-Lambertz, G., Monzalvo, K., \& Dehaene, S. (2018). The emergence of the visual word form: Longitudinal evolution of category-specific ventral visual areas during reading acquisition. PLOS Biology, 16(3), e2004103.

https://doi.org/10.1371/journal.pbio.2004103

Epstein, R., \& Kanwisher, N. (1998). A cortical representation of the local visual environment. Nature, 392(6676), 598-601. https://doi.org/10.1038/33402

Essen, D. V., Anderson, C. H., \& Felleman, D. J. (1992). Information processing in the primate visual system: An integrated systems perspective. Science, 255(5043), 419-423. https://doi.org/10.1126/science.1734518

Finzi, D., Gomez, J., Nordt, M., Rezai, A. A., Poltoratski, S., \& Grill-Spector, K. (2021). Differential spatial computations in ventral and lateral face-selective regions are scaffolded by structural connections. Nature Communications, 12(1), 2278. https://doi.org/10.1038/s41467-021-22524-2

Flechsig, P. E. (1920). Anatomie des menschlichen Gehirns und Rückenmarks auf myelogenetischer Grundlage. G. Thieme.

Glasser, M. F., Coalson, T. S., Robinson, E. C., Hacker, C. D., Harwell, J., Yacoub, E., Ugurbil, K., Andersson, J., Beckmann, C. F., Jenkinson, M., Smith, S. M., \& Van Essen, D. C. (2016). A multi-modal parcellation of human cerebral cortex. Nature, 536(7615), 171-178. https://doi.org/10.1038/nature18933 
Golarai, G., Liberman, A., Yoon, J. M. D., \& Grill-Spector, K. (2010). Differential development of the ventral visual cortex extends through adolescence. Frontiers in Human Neuroscience, 3, 80. https://doi.org/10.3389/neuro.09.080.2009

Gomez, J., Barnett, M., \& Grill-Spector, K. (2019). Extensive childhood experience with Pokémon suggests eccentricity drives organization of visual cortex. Nature Human Behaviour, 3(6), 611-624. https://doi.org/10.1038/s41562-019-0592-8

Gomez, J., Pestilli, F., Witthoft, N., Golarai, G., Liberman, A., Poltoratski, S., Yoon, J., \& GrillSpector, K. (2015). Functionally Defined White Matter Reveals Segregated Pathways in Human Ventral Temporal Cortex Associated with Category-Specific Processing. Neuron, 85(1), 216-227. https://doi.org/10.1016/j.neuron.2014.12.027

Grill-Spector, K., \& Weiner, K. S. (2014). The functional architecture of the ventral temporal cortex and its role in categorization. Nature Reviews Neuroscience, 15(8), 536-548. https://doi.org/10.1038/nrn3747

Grotheer, M., Kubota, E., \& Grill-Spector, K. (2021). Establishing the functional relevancy of white matter connections in the visual system and beyond. Brain Structure and Function. https://doi.org/10.1007/s00429-021-02423-4

Grotheer, M., Rosenke, M., Wu, H., Kular, H., Querdasi, F. R., Natu, V., Yeatman, J. D., \& GrillSpector, K. (2021). Catch me if you can: Least myelinated white matter develops fastest during early infancy. BioRxiv, 2021.03.29.437583.

https://doi.org/10.1101/2021.03.29.437583 
Grotheer, M., Yeatman, J., \& Grill-Spector, K. (2021). White matter fascicles and cortical microstructure predict reading-related responses in human ventral temporal cortex. Neurolmage, 227, 117669. https://doi.org/10.1016/j.neuroimage.2020.117669

Grotheer, M., Zhen, Z., Lerma-Usabiaga, G., \& Grill-Spector, K. (2019). Separate lanes for adding and reading in the white matter highways of the human brain. Nature Communications, 10(1), 3675. https://doi.org/10.1038/s41467-019-11424-1

Gschwind, M., Pourtois, G., Schwartz, S., Van De Ville, D., \& Vuilleumier, P. (2012). Whitematter connectivity between face-responsive regions in the human brain. Cerebral Cortex (New York, N.Y.: 1991), 22(7), 1564-1576.

https://doi.org/10.1093/cercor/bhr226

Hasson, U., Levy, I., Behrmann, M., Hendler, T., \& Malach, R. (2002). Eccentricity bias as an organizing principle for human high-order object areas. Neuron, 34(3), 479-490. https://doi.org/10.1016/s0896-6273(02)00662-1

Heil, P., Scheich, H., Budinger, E., \& Konig, R. (Eds.). (2005). The Auditory Cortex: A Synthesis of Human and Animal Research. Psychology Press. https://doi.org/10.4324/9781410613066

Johansen-Berg, H., Behrens, T. E. J., Robson, M. D., Drobnjak, I., Rushworth, M. F. S., Brady, J. M., Smith, S. M., Higham, D. J., \& Matthews, P. M. (2004). Changes in connectivity profiles define functionally distinct regions in human medial frontal cortex. Proceedings of the National Academy of Sciences, 101(36), 13335-13340.

https://doi.org/10.1073/pnas.0403743101 
Kanwisher, N. (2010). Functional specificity in the human brain: A window into the functional architecture of the mind. Proceedings of the National Academy of Sciences, 107(25), 11163-11170. https://doi.org/10.1073/pnas.1005062107

Kanwisher, N., McDermott, J., \& Chun, M. M. (1997). The Fusiform Face Area: A Module in Human Extrastriate Cortex Specialized for Face Perception. 10.

Kay, K. N., \& Yeatman, J. D. (2017). Bottom-up and top-down computations in word- and faceselective cortex. ELife, 6, e22341. https://doi.org/10.7554/eLife.22341

Klein, J. C., Behrens, T. E. J., Robson, M. D., Mackay, C. E., Higham, D. J., \& Johansen-Berg, H. (2007). Connectivity-based parcellation of human cortex using diffusion MRI: Establishing reproducibility, validity and observer independence in BA 44/45 and SMA/pre-SMA. Neurolmage, 34(1), 204-211. https://doi.org/10.1016/j.neuroimage.2006.08.022

Kosakowski, H., Cohen, M., Takahashi, A., Keil, B., Kanwisher, N., \& Saxe, R. (2021). Selective Responses to Faces, Scenes, and Bodies in the Ventral Visual Pathway of Infants. PsyArXiv. https://doi.org/10.31234/osf.io/7hqcu

Kuznetsova, A., Brockhoff, P. B., \& Christensen, R. H. B. (2017). ImerTest Package: Tests in Linear Mixed Effects Models. Journal of Statistical Software, 82, 1-26. https://doi.org/10.18637/jss.v082.i13

Lerma-Usabiaga, G., Carreiras, M., \& Paz-Alonso, P. M. (2018). Converging evidence for functional and structural segregation within the left ventral occipitotemporal cortex in reading. Proceedings of the National Academy of Sciences, 115(42), E9981-E9990. https://doi.org/10.1073/pnas.1803003115 
Levy, I., Hasson, U., Avidan, G., Hendler, T., \& Malach, R. (2001). Center-periphery organization of human object areas. Nature Neuroscience, 4(5), 533-539. https://doi.org/10.1038/87490

Li, J., Osher, D. E., Hansen, H. A., \& Saygin, Z. M. (2020). Innate connectivity patterns drive the development of the visual word form area. Scientific Reports, 10(1), 18039. https://doi.org/10.1038/s41598-020-75015-7

Luke, S. G. (2017). Evaluating significance in linear mixed-effects models in R. Behavior Research Methods, 49(4), 1494-1502. https://doi.org/10.3758/s13428-016-0809-y

Mahon, B. Z., \& Caramazza, A. (2011). What drives the organization of object knowledge in the brain? Trends in Cognitive Sciences, 15(3), 97-103. https://doi.org/10.1016/j.tics.2011.01.004

Mezer, A., Yeatman, J. D., Stikov, N., Kay, K. N., Cho, N., Dougherty, R. F., Perry, M. L., Parvizi, J., Hua, L. H., Butts-Pauly, K., \& Wandell, B. (2013). Quantifying the local tissue volume and composition in individual brains with MRI. Nature Medicine, 19(12), 1667-1672. https://doi.org/10.1038/nm.3390

Morosan, P., Rademacher, J., Gallagher, N. P.-, \& Zilles, K. (2005). ANATOMICAL ORGANIZATION OF THE HUMAN AUDITORY CORTEX: CYTOARCHITECTURE AND TRANSMITTER RECEPTORS. In The Auditory Cortex. Psychology Press.

Nordt, M., Gomez, J., Natu, V., Jeska, B., Barnett, M., \& Grill-Spector, K. (2019). Learning to Read Increases the Informativeness of Distributed Ventral Temporal Responses. Cerebral Cortex, 29(7), 3124-3139. https://doi.org/10.1093/cercor/bhy178 
Nordt, M., Gomez, J., Natu, V. S., Rezai, A. A., Finzi, D., Kular, H., \& Grill-Spector, K. (2021). Cortical recycling in high-level visual cortex during childhood development. Nature Human Behaviour, 1-12. https://doi.org/10.1038/s41562-021-01141-5

Osher, D. E., Saxe, R. R., Koldewyn, K., Gabrieli, J. D. E., Kanwisher, N., \& Saygin, Z. M. (2016). Structural Connectivity Fingerprints Predict Cortical Selectivity for Multiple Visual Categories across Cortex. Cerebral Cortex, 26(4), 1668-1683. https://doi.org/10.1093/cercor/bhu303

Peelen, M. V., \& Downing, P. E. (2005). Selectivity for the human body in the fusiform gyrus. Journal of Neurophysiology, 93(1), 603-608. https://doi.org/10.1152/jn.00513.2004

Petersen, S. E., Fox, P. T., Posner, M. I., Mintun, M., \& Raichle, M. E. (1988). Positron emission tomographic studies of the cortical anatomy of single-word processing. Nature, 331(6157), 585-589. https://doi.org/10.1038/331585a0

Pyles, J. A., Verstynen, T. D., Schneider, W., \& Tarr, M. J. (2013). Explicating the Face Perception Network with White Matter Connectivity. PLOS ONE, 8(4). https://doi.org/10.1371/journal.pone.0061611

Ratan Murty, N. A., Teng, S., Beeler, D., Mynick, A., Oliva, A., \& Kanwisher, N. (2020). Visual experience is not necessary for the development of face-selectivity in the lateral fusiform gyrus. Proceedings of the National Academy of Sciences, 117(37), 2301123020. https://doi.org/10.1073/pnas.2004607117

Rosenke, M., Weiner, K. S., Barnett, M. A., Zilles, K., Amunts, K., Goebel, R., \& Grill-Spector, K. (2018). A cross-validated cytoarchitectonic atlas of the human ventral visual stream. Neurolmage, 170, 257-270. https://doi.org/10.1016/j.neuroimage.2017.02.040 
Saygin, Z. M., Osher, D. E., Koldewyn, K., Reynolds, G., Gabrieli, J. D. E., \& Saxe, R. R. (2011). Anatomical connectivity patterns predict face selectivity in the fusiform gyrus. Nature Neuroscience, 15(2), 321-327. https://doi.org/10.1038/nn.3001

Saygin, Z. M., Osher, D. E., Norton, E. S., Youssoufian, D. A., Beach, S. D., Feather, J., Gaab, N., Gabrieli, J. D. E., \& Kanwisher, N. (2016). Connectivity precedes function in the development of the visual word form area. Nature Neuroscience, 19(9), 1250-1255. https://doi.org/10.1038/nn.4354

Shipp, S. (2003). The functional logic of cortico-pulvinar connections. Philosophical Transactions of the Royal Society of London. Series B: Biological Sciences, 358(1438), 1605-1624. https://doi.org/10.1098/rstb.2002.1213

Siegel, M., Donner, T. H., \& Engel, A. K. (2012). Spectral fingerprints of large-scale neuronal interactions. Nature Reviews Neuroscience, 13(2), 121-134. https://doi.org/10.1038/nrn3137

Smith, R. E., Tournier, J.-D., Calamante, F., \& Connelly, A. (2012). Anatomically-constrained tractography: Improved diffusion MRI streamlines tractography through effective use of anatomical information. Neurolmage, 62(3), 1924-1938. https://doi.org/10.1016/j.neuroimage.2012.06.005

Song, S., Garrido, L., Nagy, Z., Mohammadi, S., Steel, A., Driver, J., Dolan, R. J., Duchaine, B., \& Furl, N. (2015). Local but not long-range microstructural differences of the ventral temporal cortex in developmental prosopagnosia. Neuropsychologia, 78, 195-206. https://doi.org/10.1016/j.neuropsychologia.2015.10.010 
Sporns, O., Tononi, G., \& Kötter, R. (2005). The human connectome: A structural description of the human brain. PLoS Computational Biology, 1(4), e42. https://doi.org/10.1371/journal.pcbi.0010042

Stigliani, A., Weiner, K. S., \& Grill-Spector, K. (2015). Temporal Processing Capacity in High-Level Visual Cortex Is Domain Specific. Journal of Neuroscience, 35(36), 12412-12424. https://doi.org/10.1523/JNEUROSCI.4822-14.2015

Striem-Amit, E., Ovadia-Caro, S., Caramazza, A., Margulies, D. S., Villringer, A., \& Amedi, A. (2015). Functional connectivity of visual cortex in the blind follows retinotopic organization principles. Brain: A Journal of Neurology, 138(Pt 6), 1679-1695. https://doi.org/10.1093/brain/awv083

Takemura, H., Caiafa, C. F., Wandell, B. A., \& Pestilli, F. (2016). Ensemble Tractography. PLoS Computational Biology, 12(2), 1-22. https://doi.org/10.1371/journal.pcbi.1004692

Takemura, H., Rokem, A., Winawer, J., Yeatman, J. D., Wandell, B. A., \& Pestilli, F. (2016). A Major Human White Matter Pathway Between Dorsal and Ventral Visual Cortex. Cerebral Cortex (New York, N.Y.: 1991), 26(5), 2205-2214. https://doi.org/10.1093/cercor/bhv064

Tavor, I., Yablonski, M., Mezer, A., Rom, S., Assaf, Y., \& Yovel, G. (2014). Separate parts of occipito-temporal white matter fibers are associated with recognition of faces and places. Neurolmage, 86, 123-130. https://doi.org/10.1016/j.neuroimage.2013.07.085

Thomas, C., Avidan, G., Humphreys, K., Jung, K., Gao, F., \& Behrmann, M. (2009). Reduced structural connectivity in ventral visual cortex in congenital prosopagnosia. Nature Neuroscience, 12(1), 29-31. https://doi.org/10.1038/nn.2224 
Tournier, J. D., Calamante, F., \& Connelly, A. (2012). MRtrix: Diffusion tractography in crossing fiber regions. International Journal of Imaging Systems and Technology, 22(1), 53-66. https://doi.org/10.1002/ima.22005

Tournier, J. D., Smith, R., Raffelt, D., Tabbara, R., Dhollander, T., Pietsch, M., Christiaens, D., Jeurissen, B., Yeh, C. H., \& Connelly, A. (2019). MRtrix3: A fast, flexible and open software framework for medical image processing and visualisation. Neurolmage, 202(January), 116137. https://doi.org/10.1016/j.neuroimage.2019.116137

van den Hurk, J., Van Baelen, M., \& Op de Beeck, H. P. (2017). Development of visual category selectivity in ventral visual cortex does not require visual experience. Proceedings of the National Academy of Sciences, 114(22), E4501-E4510. https://doi.org/10.1073/pnas.1612862114

Wakana, S., Jiang, H., Nagae-Poetscher, L. M., van Zijl, P. C. M., \& Mori, S. (2004). Fiber tractbased atlas of human white matter anatomy. Radiology, 230(1), 77-87. https://doi.org/10.1148/radiol.2301021640

Wandell, B. A., \& Yeatman, J. D. (2013). Biological development of reading circuits. Current Opinion in Neurobiology, 23(2), 261-268. https://doi.org/10.1016/j.conb.2012.12.005

Weiner, K. S., Barnett, M. A., Lorenz, S., Caspers, J., Stigliani, A., Amunts, K., Zilles, K., Fischl, B., \& Grill-Spector, K. (2017). The Cytoarchitecture of Domain-specific Regions in Human High-level Visual Cortex. Cerebral Cortex, 27(1), 146-161. https://doi.org/10.1093/cercor/bhw361

Weiner, K. S., Barnett, M., Witthoft, N., Golarai, G., Stigliani, A., Kay, K. N., Gomez, J., Natu, V. S., Amunts, K., Zilles, K., \& Grill-Spector, K. (2018). Defining the most probable location of 
the parahippocampal place area using cortex-based alignment and cross-validation.

Neurolmage, 170, 373-384. https://doi.org/10.1016/j.neuroimage.2017.04.040

Weiner, K. S., Golarai, G., Caspers, J., Chuapoco, M. R., Mohlberg, H., Zilles, K., Amunts, K., \&

Grill-Spector, K. (2014). The mid-fusiform sulcus: A landmark identifying both

cytoarchitectonic and functional divisions of human ventral temporal cortex.

Neurolmage, 84, 453-465. https://doi.org/10.1016/j.neuroimage.2013.08.068

Weiner, K. S., Jonas, J., Gomez, J., Maillard, L., Brissart, H., Hossu, G., Jacques, C., Loftus, D., Colnat-Coulbois, S., Stigliani, A., Barnett, M. A., Grill-Spector, K., \& Rossion, B. (2016).

The Face-Processing Network Is Resilient to Focal Resection of Human Visual Cortex. Journal of Neuroscience, 36(32), 8425-8440. https://doi.org/10.1523/JNEUROSCI.450915.2016

Weiner, K. S., Yeatman, J. D., \& Wandell, B. A. (2017). The posterior arcuate fasciculus and the vertical occipital fasciculus. Cortex; a Journal Devoted to the Study of the Nervous System and Behavior, 97, 274-276. https://doi.org/10.1016/j.cortex.2016.03.012

White, A. L., Palmer, J., Boynton, G. M., \& Yeatman, J. D. (2019). Parallel spatial channels converge at a bottleneck in anterior word-selective cortex. Proceedings of the National Academy of Sciences, 116(20), 10087-10096. https://doi.org/10.1073/pnas.1822137116

Yeatman, J. D., Dougherty, R. F., Myall, N. J., Wandell, B. A., \& Feldman, H. M. (2012). Tract Profiles of White Matter Properties: Automating Fiber-Tract Quantification. PLOS ONE, 7(11), e49790. https://doi.org/10.1371/journal.pone.0049790 
Yeatman, J. D., Rauschecker, A. M., \& Wandell, B. A. (2013). Anatomy of the visual word form area: Adjacent cortical circuits and long-range white matter connections. Brain and Language, 125(2), 146-155. https://doi.org/10.1016/j.bandl.2012.04.010

Yeatman, J. D., Weiner, K. S., Pestilli, F., Rokem, A., Mezer, A., \& Wandell, B. A. (2014). The vertical occipital fasciculus: A century of controversy resolved by in vivo measurements. Proceedings of the National Academy of Sciences, 111(48), E5214-E5223. https://doi.org/10.1073/pnas.1418503111

Zeki, S., \& Shipp, S. (1988). The functional logic of cortical connections. Nature, 335(6188), 311317. https://doi.org/10.1038/335311a0

Zilles, K. (2018). Brodmann: A pioneer of human brain mapping-his impact on concepts of cortical organization. Brain, 141(11), 3262-3278. https://doi.org/10.1093/brain/awy273 